\title{
Classification and Selection of Cellular Materials in Mechanical Design: Engineering and Biomimetic Approaches
}

\author{
Dhruv Bhate ${ }^{1, *}$, Clint A. Penick ${ }^{2}$, Lara A. Ferry ${ }^{3}$ and Christine Lee ${ }^{4}$ \\ 1 The Polytechnic School, Ira A. Fulton Schools of Engineering, Arizona State University, Mesa, \\ AZ 85212-6300, USA \\ 2 The Biomimicry Center, Arizona State University, Tempe, AZ 85287-3505, USA; clint.penick@asu.edu \\ 3 New College of Interdisciplinary Arts and Sciences, School of Mathematical \& Natural Sciences, \\ Arizona State University, Glendale, AZ 85306-4908, USA; lara.ferry@asu.edu \\ 4 Herberger Institute of Design and Arts, Arizona State University, Tempe, AZ 85287-2102, USA; \\ christinelee@asu.edu \\ * Correspondence: dhruv.bhate@asu.edu; Tel.: +1-480-727-1332
}

Received: 24 December 2018; Accepted: 13 March 2019; Published: 19 March 2019

\begin{abstract}
Recent developments in design and manufacturing have greatly expanded the design space for functional part production by enabling control of structural details at small scales to inform behavior at the whole-structure level. This can be achieved with cellular materials, such as honeycombs, foams and lattices. Designing structures with cellular materials involves answering an important question: What is the optimum unit cell for the application of interest? There is currently no classification framework that describes the spectrum of cellular materials, and no methodology to guide the designer in selecting among the infinite list of possibilities. In this paper, we first review traditional engineering methods currently in use for selecting cellular materials in design. We then develop a classification scheme for the different types of cellular materials, dividing them into three levels of design decisions: tessellation, element type and connectivity. We demonstrate how a biomimetic approach helps a designer make decisions at all three levels. The scope of this paper is limited to the structural domain, but the methodology developed here can be extended to the design of components in thermal, fluid, optical and other areas. A deeper purpose of this paper is to demonstrate how traditional methods in design can be combined with a biomimetic approach.
\end{abstract}

Keywords: cellular materials; biomimicry; biomimetics; bio-inpsiration; design principles; honeycombs; foams; lattices

\section{Introduction}

The material world can, from a design standpoint, be divided into form (the bounding shape of a structure) and pattern (the constituents of this form) [1]. Cellular materials are essentially patterns that may be best defined in contrast to their homogeneous counterparts in that they are heterogeneous materials that have the following two key requirements:

- A Unit Cell: Most cellular materials are defined by a unit cell that is some combination of material and space. At its limit, a homogeneous material may be said to be a cellular material with a fully dense unit cell.

- Repetition: The unit cell is repeated in space to create the larger structure or surface-the resulting pattern need not be regular and may include more than one type of unit cell. 
Cellular materials offer advantages that cannot be easily availed of from homogeneous structures, such as the ability to locally tune properties and to add multi-functionality to component parts [2]. This has been exploited in a wide range of structural, thermal, fluid and biomedical applications, listed in Figure 1, adapted from a design guide for metal foams [3].

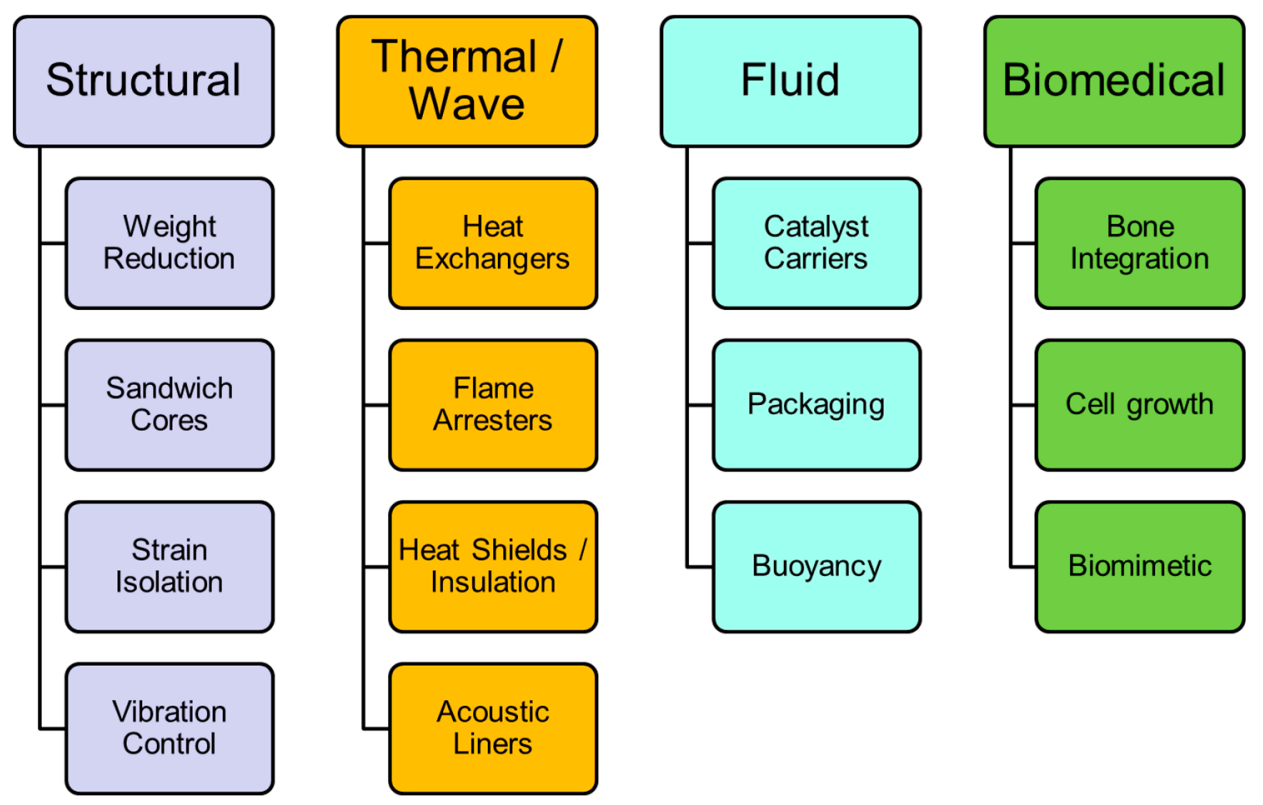

Figure 1. Applications for cellular structures that can leverage their special properties to enhance functional performance (adapted from a design guide on metal foams [3]).

Recent developments in manufacturing, such as with Additive Manufacturing (AM) technologies, and design software, have now made it significantly easier to design and manufacture geometries that were hitherto cost prohibitive, or simply not feasible to fabricate. While the advantages of designing with cellular materials are increasingly apparent, several challenges limit the widespread implementation of additively manufactured cellular materials in functional parts. One of these challenges is the lack of a methodology for designing structures with cellular materials to optimize function, including approaches to assess which cellular pattern might work best for a specific application, which is the focus of this review. We identify traditional engineering approaches and propose combining them with biomimicry for identifying optimal strategies when designing with cellular materials. The former approaches have been developed by researchers over the past century and are well documented in textbooks and literature, which are briefly reviewed in the next section.

Over 3.95 billion years of evolution [4], organisms have developed a variety of cellular geometries that conserve material usage and enhance performance under the conditions where life thrives. As early as the 1st century BC, the Roman scholar Varro postulated on the cellular design of honeycomb and its material-saving properties [5], and in 1665, using microscopy, Robert Hooke speculated correctly that the behavior of cork could be attributed to its cellular structure [6]. Since this time, scientists have investigated a wide variety of cellular patterns in nature and postulated about their functions [7], summarized in Figure 2. Imaging technology has also greatly improved and now commonly includes X-ray tomography methods [8]. The similarities between Figures 1 and 2 are strongly suggestive of the fact that nature and engineers have both used cellular materials to attain similar objectives. 


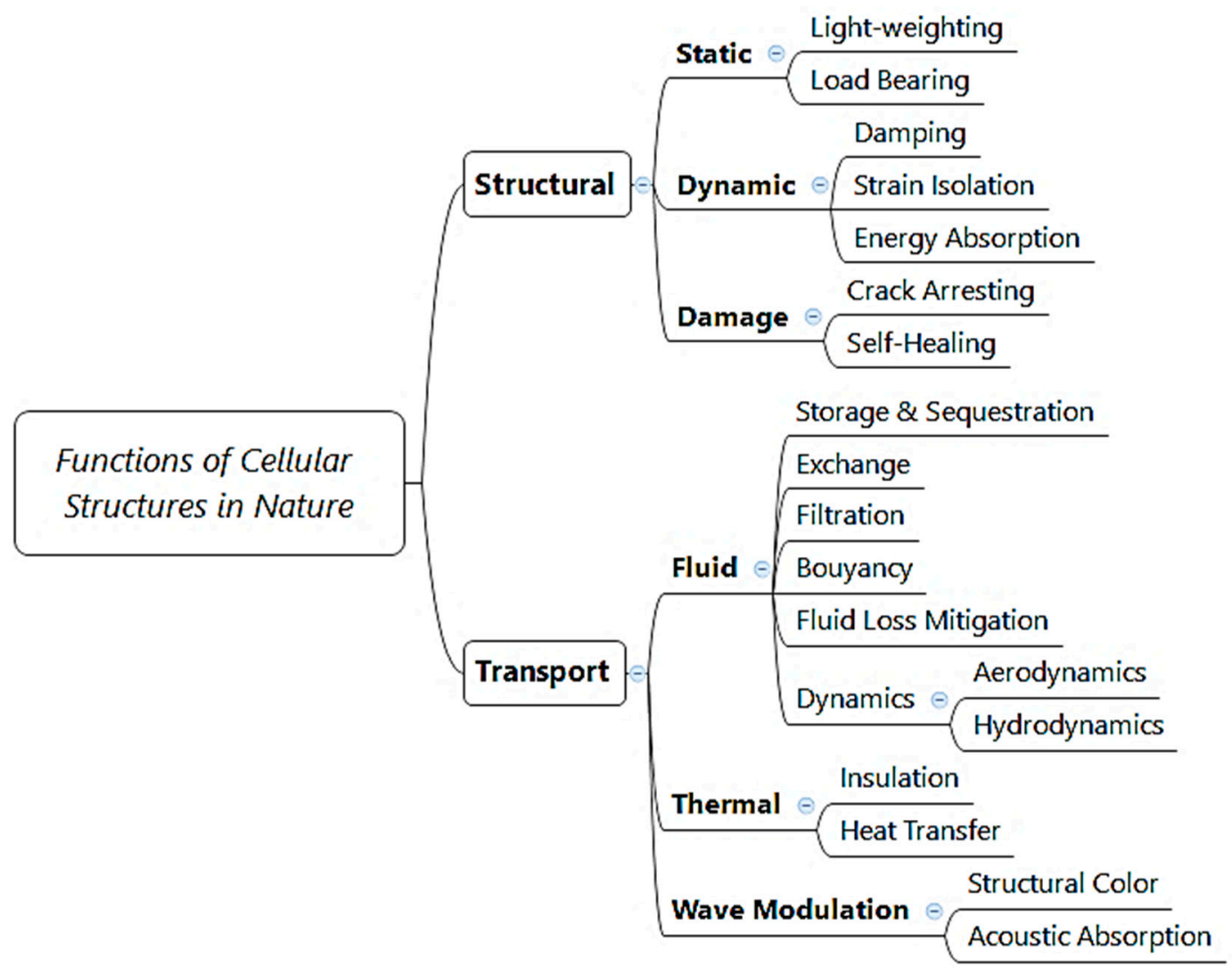

Figure 2. Functions of cellular structures in nature, following [9].

Section 2 of this paper discusses traditional engineering approaches to cellular material selection. In Section 3, we present a three-level classification scheme to aid the designer of cellular materials. Section 4 describes how a biomimetic approach can be used to guide the designer in the selection of options at all three levels of the classification scheme. Finally, in Section 5, we discuss how combining engineering and biomimetic approaches can help a designer traverse the design space and select an optimum cellular shape.

\section{Engineering Approaches to Cellular Material Selection}

Broadly speaking, there are three types of approaches used by engineers to select an appropriate unit cell for a specific application. The first is analytical in nature, where first principles are invoked to represent behavior in the form of mathematical models. The second approach is empirical in nature, where engineers rely on experimental or computational data to develop predictive models or compare one material against another. The third approach involves the use of computational tools where the material design is an emergent property. In practice, a combination of these approaches may be most useful, especially in more complex, multi-objective contexts.

Before examining these approaches in more detail, however, it is useful to ask: what is the figure of merit that is being compared? For the three structural properties of interest in this paper (stiffness, strength and toughness), what is the appropriate metric? The next section first examines the different ways this metric can be estimated, and then discusses the analytical and empirical methods that may be used to compare shapes with these metrics in mind.

\subsection{Figures of Merit}

To select a homogeneous material (one that is not cellular), one simply looks up a material property chart such as the one shown in Figure 3, and selects, for example, a Titanium alloy over an Aluminum one if higher stiffness is desired. However, this is not as straightforward when it comes to cellular 
materials, since the observed property is a combination of both composition (material) and design (cellular structure). A chart such as the one in Figure 3 provides little granularity regarding the relative contributions of each, limiting the designer's ability to make an insightful selection. An additional complication is that cellular materials can respond differently depending on the specific loading conditions - a specific shape that has a compressive strength may not be optimum under bending, for example.

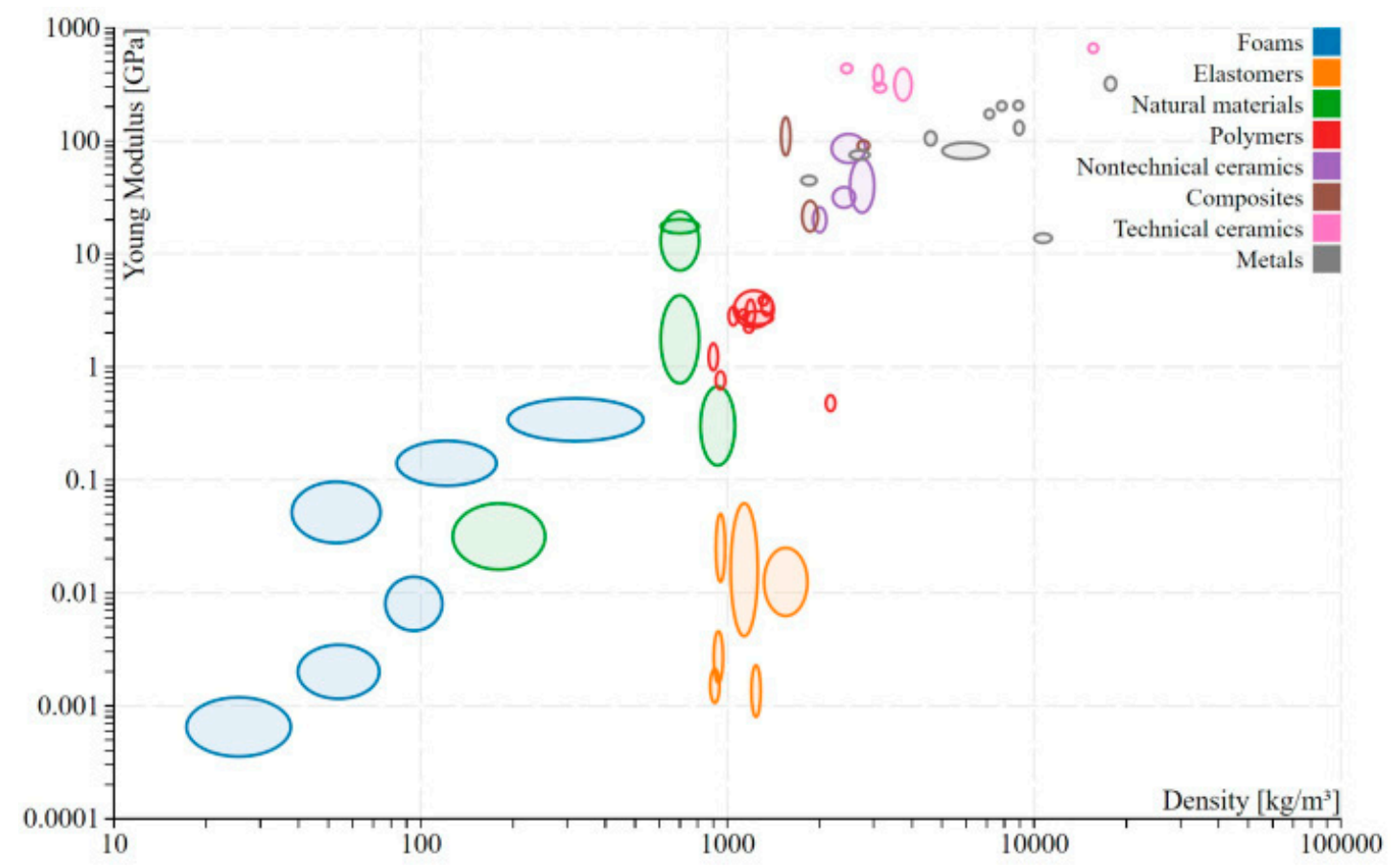

Figure 3. The "Ashby" plot for Young's modulus (Y-axis) and density (X-axis) is an effective tool in material selection but does not isolate geometry from composition for cellular materials (Attr: Nicoguaro, Wikimedia Commons).

The Ashby plot in Figure 3 can be used for cellular materials with the use of the effective property, which is estimated just as properties of homogeneous materials would, typically through experimental studies [6]. To address integration of this cellular material into a more realistic loading environment, materials indices introduced by Ashby [10] can be used. These indices are a function of the structural geometry (such as tie, beam, column or panel), the objective of interest (weight, cost) and the constraints prescribed (stiffness, strength, buckling load). Given these three pieces of information, a material index can be specified that enables selection among a range of materials. For strength-limited designs for example, with the objective of minimizing mass, the index becomes [10]:

$$
\begin{aligned}
& \sigma_{y} / \rho \text { for a tie (rod) structure } \\
& \left(\sigma_{y}\right)^{0.5} / \rho \text { for a panel structure }
\end{aligned}
$$

where $\sigma_{y}$ is the yield strength, and $\rho$ is the density of the material. The designer would seek materials that maximize these indices-and select the highest one available (all other things being equal). For cellular materials, these indices would be composed of effective properties but otherwise the process is identical. Material indices, while useful in selection for a given material composition, do not enable an isolation of geometry from composition. Another critique of these indices is that they often lack physical insight and possess unfamiliar units that can be difficult to interpret, depending on the specific index under consideration. 
Another dimensionless metric called the relative effective property is simply expressed as the effective property of interest divided (normalized) by the same property but for the material that constitutes the cellular structure. Therefore, to cite one example, instead of comparing just the effective moduli $E^{*}$ of two cellular materials, this metric would compare their relative effective moduli:

$$
\text { Relative Effective Modulus }=E^{*} / E_{s}
$$

where $E_{s}$ represents the effective modulus of the solid material from which the cellular material is composed. Normalizing the property being maximized (or minimized) by its equivalent bulk material property isolates contributions of composition. A disadvantage of using the relative effective property is that it does not address the material utilization (density) aspects in the figure of merit itself. For this purpose, a metric that includes the benefits of normalization but also combines it with a consideration of relative density, is needed.

Berger et al. [11] introduced a measure of geometric efficiency of the cellular material design, which is independent of the composition of the material used to create it. For effective modulus of a cellular material, for example [11]:

$$
\text { Geometric Efficiency Index }=\frac{E^{*} / E_{s}}{\rho^{*} / \rho_{s}}
$$

where $E^{*}$ and $\rho^{*}$ represent the effective modulus and density of the cellular material, while $E_{S}$ and $\rho_{s}$ represent their bulk material counterparts. The power of such a metric is that it allows a designer to answer a question such as what is the stiffest isotropic cellular material design. Traditionally, in material selection handbooks, this answer can be obtained by a study of plots of modulus vs density, where material indices are prescribed depending on the objective and constraint of interest. However, as mentioned previously, this approach does not isolate the contributions of material from geometry. Berger et al. [11] examined different metamaterials with the aim of identifying ones near the theoretical limit for elastic stiffness.

In summary, if composition is fixed and loading conditions are well characterized by standard testing procedures, the effective property (e.g., $E^{*}$ ) may be adequate to compare cellular material designs and select an appropriate cellular geometry. If composition is also allowed to vary, the property of interest needs to be normalized with its bulk material counterpart (i.e., $E^{*} / E^{s}$ ). If the objective with cellular material selection is to achieve the maximum performance with the lowest mass, cellular materials should be compared using the geometric efficiency index.

\subsection{Maxwell's Stability Criterion}

The 1800s were a period of expanding construction, and one of the concerns was developing theories for how to design structures. Maxwell pioneered the field of the mathematical theory of structural rigidity and 1864 published a paper developing a simple equation that discusses stability in the context of rigid truss structures [12]—conveniently, this is also of relevance to cellular materials when discussing lattice structures. The results developed by Maxwell have come to be known as Maxwell's stability criterion, and it involves the computation of a metric $M$ for a lattice-like structure with $b$ struts and $j$ joints as follows [12]:

$$
\begin{gathered}
\text { In 2D structures: } M=b-2 j+3 \text { and, } \\
\text { In 3D structures: } M=b-3 j+6 .
\end{gathered}
$$

Per Maxwell's criterion, and assuming the joints are locked, the following three scenarios are possible, which are graphically shown for a 2D structure in Figure 4:

- If $M<0$, the structure is under-constrained

- If $M=0$, the structure is a rigid framework 
- If $M>0$, the structure is over-constrained

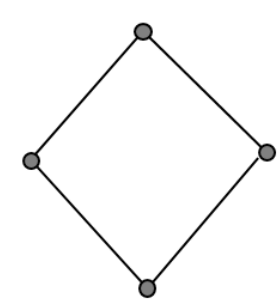

$\mathrm{M}<0$

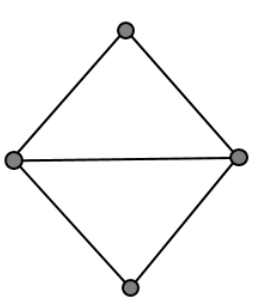

$M=0$

- Stretch dominated

- Stiff structures

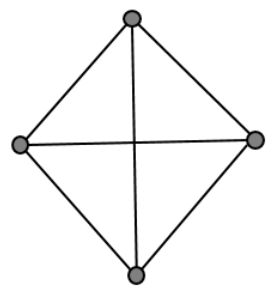

$\mathrm{M}>0$
- Bending dominated

- Compliant structures
- Over-constrained design

- Internal stresses

Figure 4. The relationship between Maxwell's stability criterion and stretch- and bending-dominated structures [12,13].

The rigidity of the frame structure has a direct relationship to its behavior in a lattice. This connection was made in the context of foam topology and demonstrated for a range of different shapes specifically in the context of determining if a structure was stretch- or bending-dominated [13]. As shown in Figure 4, under-constrained structures are bending dominated while rigid structures are stretch dominated. What this means from a design standpoint is that if stiff structures are desired, lattice structures that have $M=0$ are ideal for the task. If compliant structures are sought, structures with $M<0$ are more appropriate. To quote the cited paper, "the modulus and initial yield strength of a stretching-dominated cellular solid are much greater than those of a bending-dominated cellular material of the same relative density." [13].

The main advantage of using Maxwell's stability criterion in the context of cellular materials is the significant insight it provides given its relative simplicity. However, it is also very limited to beam-based structures, and only gives information into the expected nature of deformation. This deformation mode is also limited to the very specific direction of uniaxial, in-plane loading. Maxwell's stability criterion also does not provide insight into the role of the base material and its property (it assumes rigid, infinitely stiff rods), or how the dimensions of these rods influence behavior. Nonetheless, it allows us to quickly categorize lattice structures into stretch- and bending-dominated structures for such load cases, which is a crucial design consideration for structural applications.

\subsection{Relative Density Scaling Laws}

Relative density is one of the most important features of a cellular material and has been a critical figure-of-merit in the use of foams. It can be used in conjunction with property equations to make design decisions when density is of importance-in buoyancy or light-weighting applications, for example. Relative density is expressed as $\rho^{*} / \rho_{\mathrm{s}}$, where $\rho^{*}$ is the density of the cellular material, and $\rho_{\mathrm{s}}$ the density of the material of which the cellular structures are made [6]. Relative density can be calculated from the geometry of the shape and for beam-based structures is typically some function of the ratio of the thickness of the member (edge or wall) over its length $(t / l)$. For honeycombs and foams, for example, these relationships typically take the forms below, where $C_{1}, C_{2}$ and $C_{3}$ are constants [6]:

$$
\begin{gathered}
\text { Honeycomb : } \frac{\rho^{*}}{\rho_{\mathrm{s}}}=C_{1} \frac{t}{l} \\
\text { Open Cell Foam : } \frac{\rho^{*}}{\rho_{\mathrm{s}}}=C_{2}\left(\frac{t}{l}\right)^{2}
\end{gathered}
$$




$$
\text { Closed Cell Foam : } \frac{\rho^{*}}{\rho_{\mathrm{s}}}=C_{3}\left(\frac{t}{l}\right)^{3}
$$

A table of such relationships for different shapes can be found in [6]. For more complex shapes, relative density can be computed from an output of the design file used to create it, and it can also be measured experimentally. A designer is typically concerned with selecting a certain shape, not in knowing its relative density per se. This is where relationships between a certain property and the relative density are useful—these relationships are also commonly termed "Scaling Laws". Scaling laws are most well developed for making predictions on mechanical response. For effective modulus, for example, the relationship typically takes the following form, where $E^{*}$ and $E_{S}$ are effective modulus of the cellular material and of the bulk solid, respectively [6]:

$$
E^{*}=C E_{s}\left(\frac{\rho^{*}}{\rho_{s}}\right)^{n}
$$

Table 1 lists the relative densities and relative effective property equations based thereon for different cellular materials and can be used as the basis for design selection when relative density is important to the design. For example, for the same relative density, it is clear that an octet is a third as stiff under compression as a triangular honeycomb loaded out-of-plane.

Table 1. Material property scaling for some cellular materials, from [14].

\begin{tabular}{ccccc}
\hline & $\begin{array}{c}\text { Relative } \\
\text { Density } \\
\rho^{*} / \rho_{s}\end{array}$ & $\begin{array}{c}\text { Young's } \\
\text { Modulus } \\
\boldsymbol{E}^{*} / E_{s}\end{array}$ & $\begin{array}{c}\text { Compressive } \\
\text { Strength } \\
\sigma^{*} / \sigma_{s}\end{array}$ & $\begin{array}{c}\text { Fracture } \\
\text { Toughness }\end{array}$ \\
\hline Triangular & $\frac{2}{\sqrt{3}} \frac{t}{l}$ & $\frac{1}{3} \frac{\rho^{*}}{\rho_{s}}$ & $\frac{1}{3} \frac{\rho^{*}}{\rho_{s}}$ & $\sim \frac{1}{2} \frac{\rho^{*}}{\rho_{s}}$ \\
honeycomb-out-of-plane & $\frac{1}{9} \frac{\rho^{*}}{\rho_{s}}$ & $\frac{1}{3} \frac{\rho^{*}}{\rho_{s}}$ & $0.32 \frac{\rho^{*}}{\rho_{s}}$ \\
\hline
\end{tabular}

In principle, scaling laws can be developed for any property where physical principles suggest a relationship between that property and the relative density of the cellular material, by manufacturing and testing materials at a range of relative density values and then fitting an appropriate relationship to the observed datasets. This method, while empirical and likely geometry dependent, can prove to be a useful design protocol in the absence of analytically derived relationships.

It is also important to recognize that scaling laws ignore geometric detail—thus, whether the relative density is obtained using variation of parameters (changing thickness of walls and struts, for example) or through changing cell sizes as shown in Figure 5, or a combination of both strategies - these are all equivalent for the purposes of property estimation. For the effective properties discussed here, these may be reasonable strategies. For studying the failure of cellular materials, they are likely to be inadequate. 


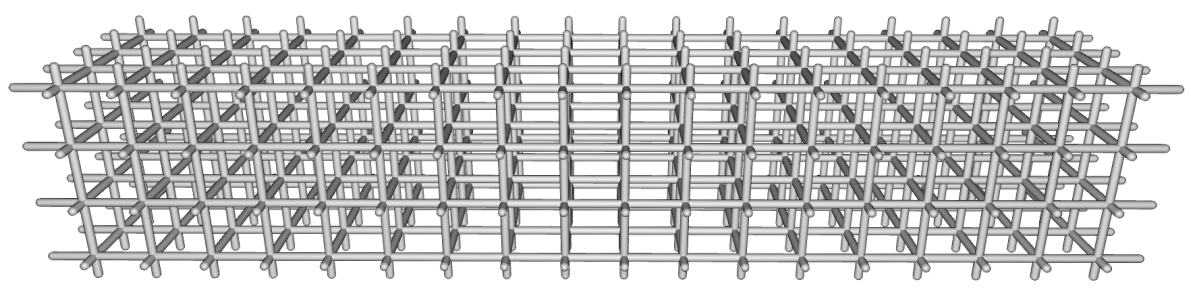

(a)

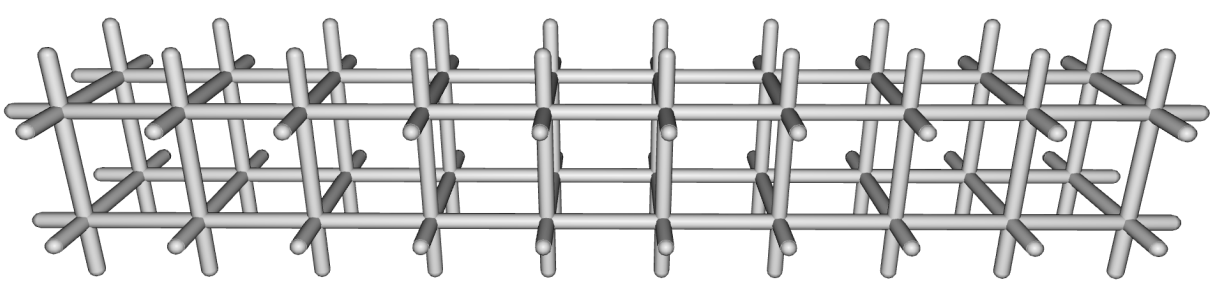

(b)

Figure 5. Two different lattice designs with identical relative density: (a) the lattice has a small cell size with a thin strut diameter, $(\mathbf{b})$ the lattice has a larger cell size with a thicker diameter.

\subsection{Empirical Methods}

The most commonly used method of selecting a particular cell shape is to test them empirically, either experimentally or computationally [15-19]. These datasets can also be used as the basis to fit the aforementioned scaling laws to experimental data [20] and derive the parameters that can then be used in design. Computational methods can also be used to study the relative merits of cellular materials in a specific loading environment. This can be demonstrated for the case of a beam bending problem. As shown in Figure 6a, this beam was simply supported on either end and had a distributed edge load applied across the top surface. The beam was then populated with several different cellular material designs, and displacement calculated, one example of which is shown in Figure $6 \mathrm{~b}$. This displacement was used to solve for an effective flexural rigidity, $(\mathrm{EI})^{*}$, where I is the area moment of inertia. Following Berger et al.'s notion of geometric efficiency [11], this quantity was normalized with respect to the flexural rigidity $E_{s} I$ of a solid beam constructed with material $E_{s}$. While the specific conclusions and differences are not relevant for the present discussion, Figure $6 \mathrm{c}$ shows how different cellular materials can be compared for a specific loading condition and one selected from a range of different options. In this specific study, for example, one may conclude that the "tet oct edge" shape is the cellular material with the highest geometric efficiency with regard to flexural rigidity, among the shapes studied. 


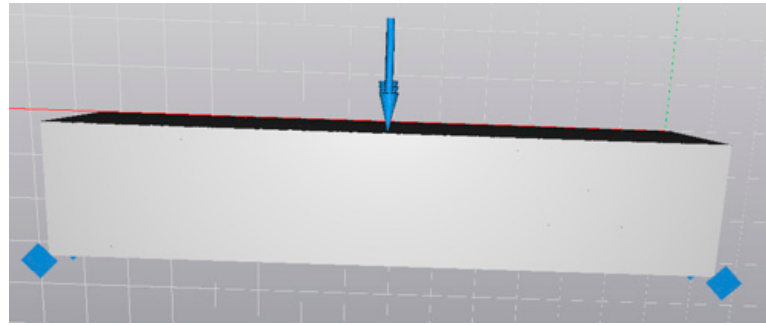

(a)

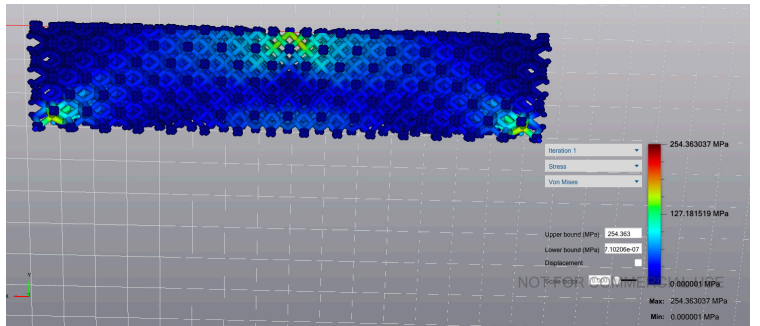

(b)

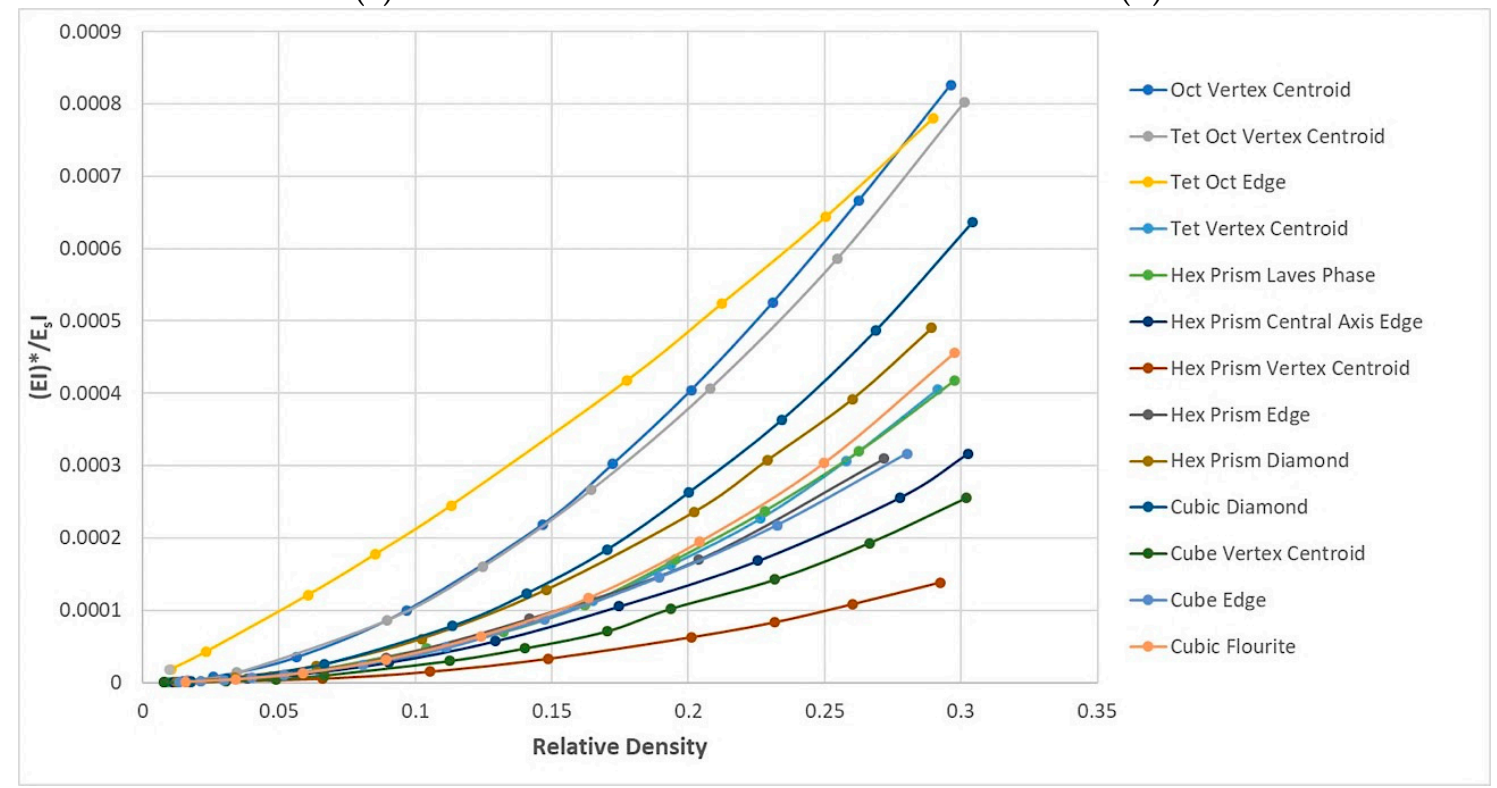

(c)

Figure 6. Empirical study conducted with nTopology's Element software [21] to identify cellular material with the highest geometric efficiency for bending stiffness: (a) model setup; (b) typical stress contours and (c) graph comparing different cell shapes across a range of relative densities.

\subsection{Simulation-Driven Cellular Material Design}

While analytical and empirical methods help narrow down the field of cellular material selection, there may be instances, particularly with regard to multi-functional design, where the optimum cellular material is not one that is readily identifiable among available choices. In fact, there is work to suggest that lattice structures may not be optimal for stiffness objectives at any length scale, including at the cellular material level [22], and therefore it is only in multi-objective scenarios that cellular materials may be worth the additional design cost. In such circumstances, a computational method based on optimizing the design of the unit cell itself may prove to be useful since it may be challenging to do so analytically, and cost-prohibitive to evaluate empirically. One of these approaches is to use a multi-scale optimization approach as described by Osanov and Guest [23] and Cadman et al. [24]. In this approach, the unit cell domain is discretized into elements which are then optimized using topology optimization methods [25]. Once a unit cell is designed, effective properties are determined. Test fields are applied to the unit cell architecture along with periodic boundary conditions to compute a response and estimate properties, typically using Finite Element Analysis (FEA), since analytical models only exist for the simplest of geometries. In the final step, inverse homogenization is used to upscale the cellular geometry to the level of the larger structure [26]. These ideas have been extended to multi-material cellular optimization as well [27]. Recent work has looked at implementing some of these ideas, inverse homogenization in particular, towards integration into commercial FEA code [28,29]. Finally, cellular automata methods have also been proposed to design materials [30] and microstructures [31], and machine learning methods are also beginning to be applied to materials 
design [32,33]. These latter methods do not require a repeated unit cell and in that sense are not within scope, but may be said to represent a specific instance of cellular material design where each cell is allowed to have an independently varying form.

\section{A New Classification of Cellular Materials}

A designer seeking to leverage cellular materials can select from, quite literally, an infinitely large list of unit cells. One way to make the selection process tractable is to classify cellular materials based on shape and then associate these classes of shapes with functional benefit. Several approaches have been developed, either explicitly or implicitly, to classify cellular materials. The most common approach follows the work done by Gibson and Ashby [6], who proposed classifying cellular materials as either honeycombs or foams. Honeycombs are defined as prismatic cellular structures, where a two-dimensional template is extruded in the third direction, and the name owes its origins to the nest constructed by the honeybee, shown in Figure 7 (left). Honeycombs, in engineering parlance, need not always be hexagonal and can assume square, triangular or irregular forms as well [6]. In contrast to a honeycomb, a foam is a three-dimensional cellular material that is typically the result of a foaming process and often has a stochastic form as a result-an example of this structure is shown in Figure 7 (right).
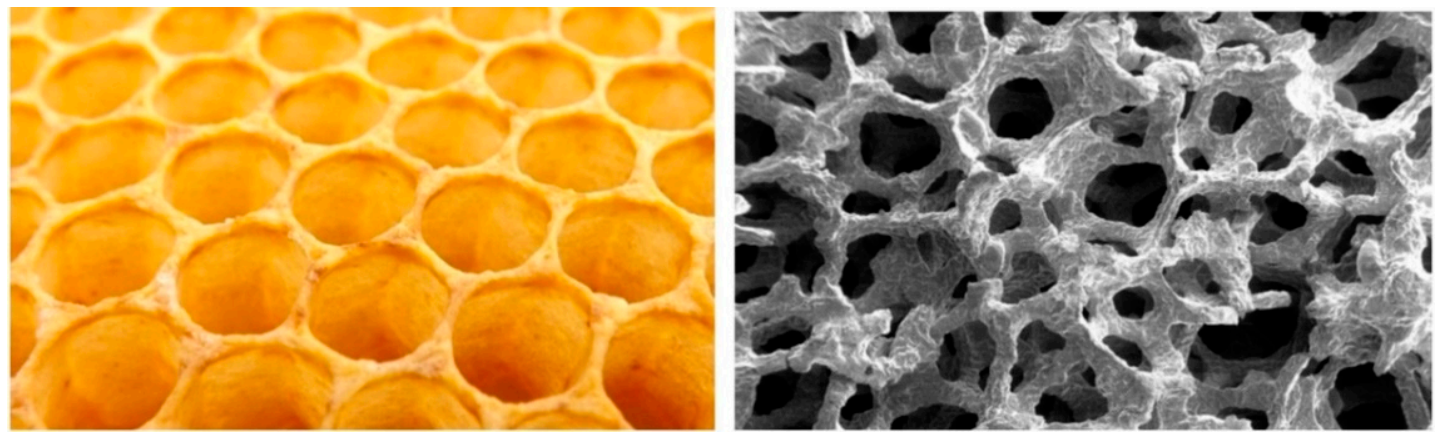

Figure 7. The bee's honeycomb (left) is an example of a prismatic cellular material. The stochastic metal foam (right) is an example of a 3D cellular material (Attr: SecretDisc, Wikimedia Commons).

Honeycombs [34] and foams [35] have dominated the landscape of engineering cellular materials over the past two decades with little need for a more formal nomenclature since these were the two classes of cellular materials that were readily manufacturable with existing technologies and therefore most in use. However, as a comprehensive classification scheme, this simplistic separation of cellular materials falls short of describing all the possibilities in the design space. Towards this end, two areas of study that deal with unit cell shapes are mathematics and crystallography. Within mathematics, the field of tessellation deals with the partitioning of space into smaller units or cells [36-38]. Crystallography, the science of crystal structure, has also developed nomenclature schemes that are amenable to the study of ordered cellular materials. Examples of these include the BCC (Body Centered Cubic) and FCC (Face Centered Cubic) crystal structures. This approach has been used as the basis for a nomenclature scheme proposed by Zok et al. [39], which is, however, limited to truss-based structures. Finally, there is at least one effort in the literature to develop design guidance for the selection of prismatic cellular materials, but this does not include three-dimensional cellular materials [40]. In this section, ideas from these sources are synthesized to develop a comprehensive classification scheme. This is also the scheme that the subsequent discussion of natural cellular materials is based on.

At the outset, it helps to think of cellular materials at the following three levels:

1. Tessellation: The compartmentalization of space into independent volumes of a certain shape

2. Elements: The use of beams and/or shells and faces within the tessellated space

3. Connectivity: The actual arrangement of the elements within the tessellated space 
Within each of these levels, a designer can choose among a range of options. These options are shown as a classification scheme in Figure 8. A designer thus must define a tessellation, specify what elements lie within the tessellation, and finally describe how these elements are connected to each other. In the following discussion, each of these design choices, represented as dots in Figure 8, are examined. In subsequent sections these choices are discussed in more detail.

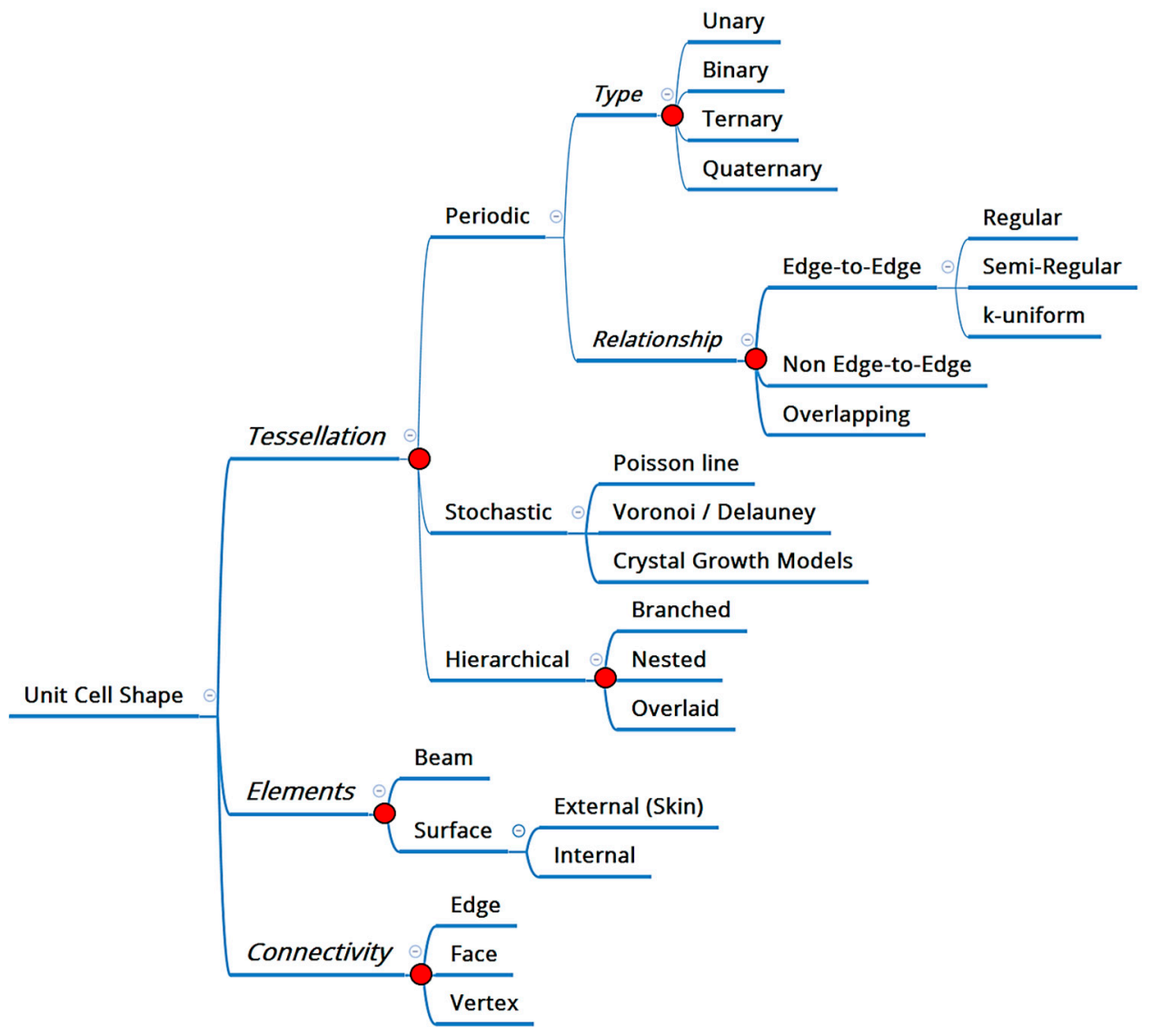

Figure 8. Cellular material classification developed by the authors from a synthesis of schemes from many domains. Each dot represents a decision point.

\subsection{Tessellation}

Designing structures with cellular materials ultimately involves filling space. From a mathematical perspective, this is a question that falls within the purview of geometry, and more specifically in the domain of tiling, or tessellation. Broadly speaking, space can either be tessellated in periodic, non-stochastic shapes (where the shape of each cell and its connectivity to others is prescribed, such as the regular hexagon honeycomb pattern), or stochastic shapes (where the shape emerges as a result of an underlying stochastic function, such as Voronoi patterns). Each of these two approaches are discussed in turn, first for the simpler case of 2-dimensional (2D) tessellation, and then for 3-dimensions (3D).

\subsubsection{Periodic Tessellation}

Attempts have been made since the time of the ancient Greeks to develop a classification scheme for tessellating space with pre-defined shapes, with approaches of the most rigor found in mathematics [36,41,42]. From a designer's perspective, periodic tessellation is about the selection of one or more polygonal (in 2D) or polyhedral (in 3D) shapes and the relationships between their connections. Thus, there are two levels of descriptors: the first is the type, and the second is the relationship. Repeating unit cell entities may be constituted of one, two, three, four or more different 
polygons/polyhedra, and accordingly are termed Unary, Binary, Tertiary and Quaternary, respectively. The relationship defines how these shapes are connected to each other. Examples of different types and relationships are shown in Figure 9 for a few different tessellation schemes.

\section{Edge-to-Edge}

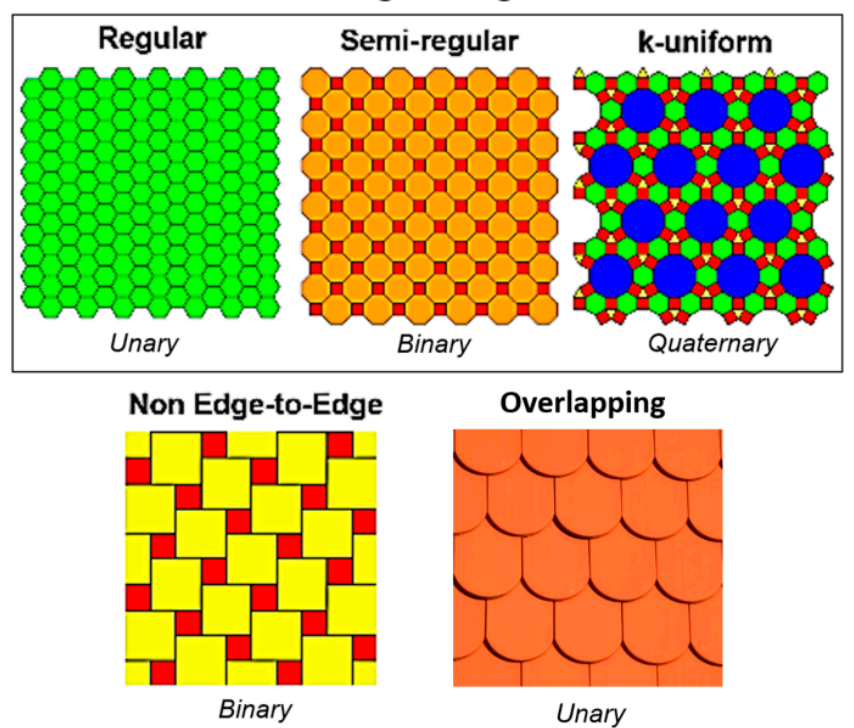

Figure 9. A selection of tessellation relationship schemes (Wikimedia Commons Attribution-Tomruen, following [36]).

The most commonly studied (and tractable) tessellations are Edge-to-Edge, which is a class of tessellations that ensures that when two polygons intersect at more than one point, they always share complete edges. This is true of the first three tessellations in Figure 9, but not of the fourth, which is a Non-Edge-to-Edge configuration, since at least one shape shares a partial edge with another. Within Edge-to-Edge tessellations, the polygons can be regular (all identical—of which there are only three permissible shapes: hexagon, square and triangle) or semi-regular (when there is more than one polygon). Focusing on the symmetry around vertices allows the introduction of a $\mathrm{k}$-uniform classification, where $\mathrm{k}$ represents broadly the different possible surroundings around a vertex. Finally, an overlapping tessellation is a relationship where unit cells may lie on top of each other with partial overlaps, much in the manner of rooftop tiling.

\subsubsection{Stochastic Tessellation}

Stochastic tessellation does not rely on the pre-supposition of one or more polygons (or polyhedral) that form an aggregate structure. Instead, stochastic tessellations emerge from an underlying function that has a random distribution specified within it. The most common way of representing stochastic tiling is using the Voronoi diagram, attributed to Ukrainian mathematician Georgy Voronoi, who defined and generalized the n-dimensional case in 1908 [37]. Other methods include using the Poisson line method and crystal growth models [37,38]. In its simplest manifestation, a Voronoi diagram emerges from a distribution of a finite number of randomly distributed points in space, around which a cell is drawn such that every point is equidistant from the lines formed at the intersections of the cells, as shown in Figure 10. 


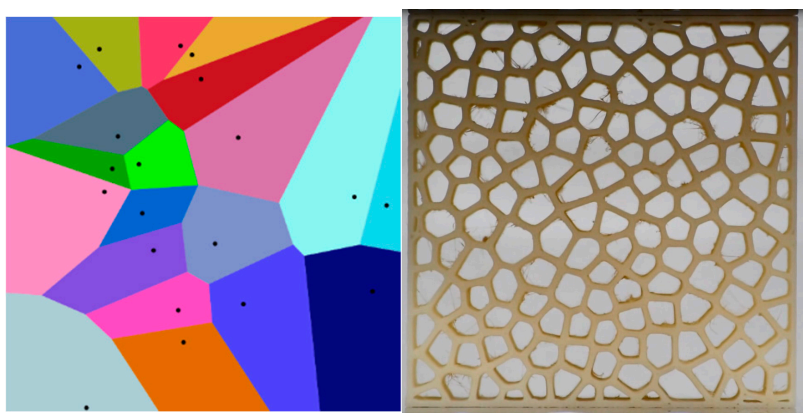

Figure 10. Voronoi diagram (left, Attribution: Wikimedia Commons, Balu Ertl), and 3D printed Voronoi prismatic structure.

\subsubsection{Hierarchical Tessellation}

A form of tessellation common in nature, but not often seen in engineering applications, emerges around a hierarchy, which can take several forms. One such form is branching, as in the case of the dragonfly wing in Figure 11a. It has been shown that a collection of so-called "primary" veins are such dominant features that they may be used to identify species [43]. Cells in this context may be seen as emerging from an overriding branching pattern.

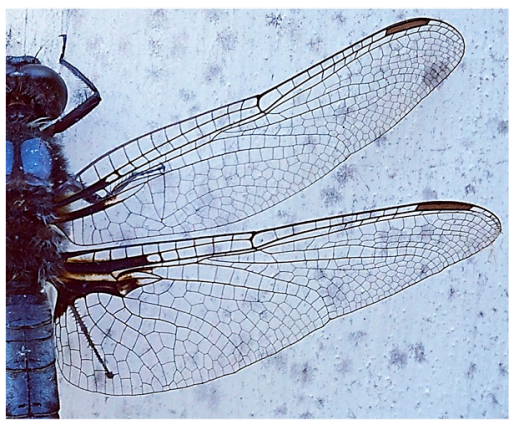

(a)

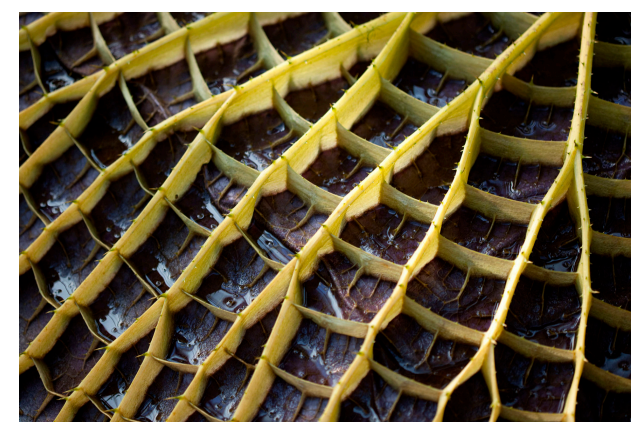

(b)

Figure 11. Branching patterns in dragonfly (a) and the Amazon water lily (b) demonstrate how tessellation can be defined in the context of branches (Attr: lily by Laitr Keiows, Wikimedia Commons).

The examples discussed so far are two-dimensional (2D), or prismatic cellular materials-what Gibson and Ashby term honeycombs [6]. These prismatic cellular materials have strong anisotropy in the out-of-plane direction, i.e., the behavior of these materials is different depending on which direction the property of interest is measured in. Thus, from an application standpoint, the 2D nature of prismatic cellular materials means that their use is most often restricted to conditions where the directionality of the environmental loads are predictable and the design can be oriented in a certain way to extract maximum benefit. Examples of this include crash panels in the automotive industry, sandwich panels in construction, and automotive radiator grilles. In all of these cases, the direction of the environmental stimulus is unidirectional and predictable - whether it be mechanical load or fluid flow. However, there are several applications where this is not the case, such as foam packaging for energy absorption, commonly used in the shipping industry, and this is a key reason for needing three-dimensional (3D) cellular materials.

\subsubsection{Extension to 3D}

For 3D cellular materials, Pearce [44] identifies 23 different space-filling polyhedra. Three of these are prismatic: the cube, triangular prism and hexagonal prism. The remaining 20 space-filling polyhedra include non-prismatic but commonly known forms, such as the tetrahedron. As before, a cellular material tessellation could be unary, binary, ternary or quaternary. Pearce suggests that 
higher levels of tessellation do not occur in nature. Table 2 lists some space-filling tessellations that have interesting properties and may form the basis of tessellation for cellular materials for designers. For a more complete study, the reader is referred to the titles by Pearce [44,45].

Table 2. Examples of 3D space-filling with non-prismatic polyhedra.

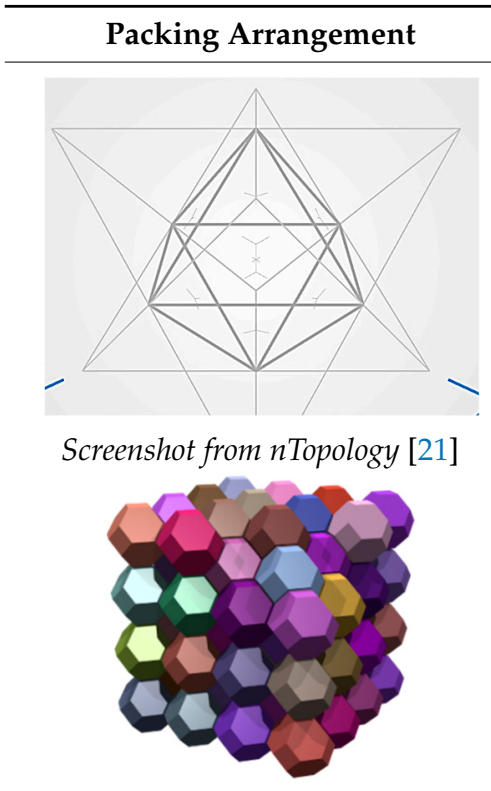

Attr: AndrewKepert, Wikimedia

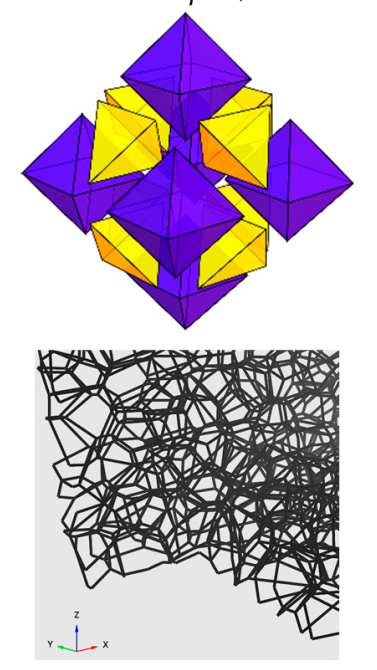

Screenshot from nTopology [21]
Unary system composed of a regular octahedron

Unary system composed only of the semi-regular truncated octahedron

Binary system composed of two regular polyhedra: the octahedron and the tetrahedron

Stochastic tessellation
One of the only regular (all faces and edges are identical), non-prismatic polyhedra that fill space by itself

One of the lowest surface area per unit volume space-filling systems (but not the lowest)

Results in the "octet" lattice structure when lattice beams are placed along each edge- $-\mathrm{a}$ commonly used structure due to its high stiffness

Voronoi tessellation in 3D

\subsection{Elements}

Tessellation is merely the first in a 3-step process to defining a 3D unit cell. The next step involves deciding what physical elements will occupy that space. These can be broken down into two types of elements: (1) beam elements, and (2) surface elements. Surface elements in turn can apply externally to the skin of a structure, or may be distributed internally within the structure itself.

\subsubsection{Beam Elements}

Cellular materials composed of beams are among the most popular choices in design and are commonly called lattice structures, or simply, lattices [46-49]. In a lattice, the length of the beam is governed by the placement of vertices in space, which is discussed in the next section. However, the beam itself has a cross-section that can take a range of shapes, including a variable 
section from one end to another. Figure 12 shows three possible cross-sections one can assign to a beam. Depending on the scales involved, such nuances may not be resolvable, but if they are, they become a design variable that can influence behavior as well as manufacturability. An example of a design variable related to beam elements is the teardrop shape in Figure 12c, which is designed to enable overhanging lattice beams to be self-supporting for processes that need support in Additive Manufacturing processes such as laser powder bed fusion [50]. Others have also looked at introducing waviness into these lattice beams to study their effects on mechanical performance [51].

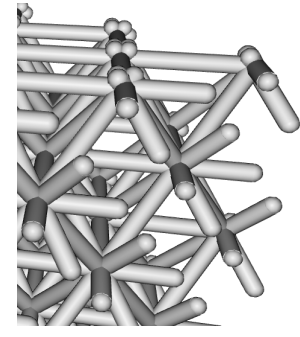

(a)

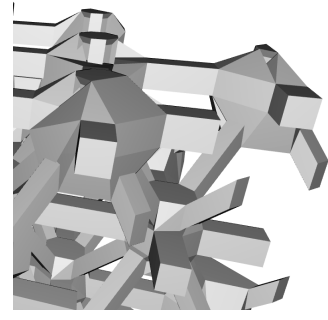

(b)

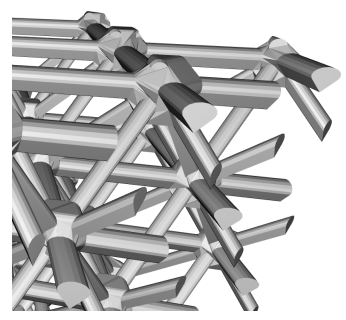

(c)

Figure 12. Different cross-sections for the beams that constitute the lattice: (a) circular section, (b) square section and (c) teardrop shape to aid in self-supporting overhangs (screenshots from nTopology's Element software) [21].

\subsubsection{Surface Elements}

An alternative to beams is the use of surfaces to construct the unit cell. Here there are two further possibilities-the use of flat surfaces or curved surfaces. An example of a flat surface cellular material and a basic minimal surface are shown in Figure 13a,b, respectively.

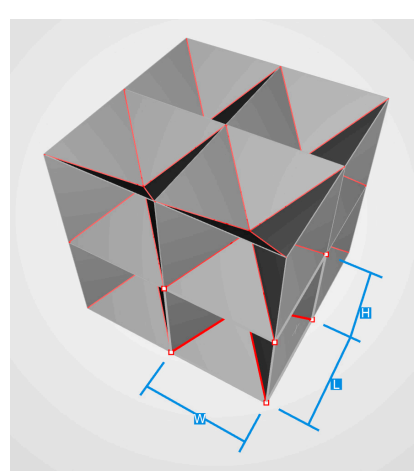

(a)

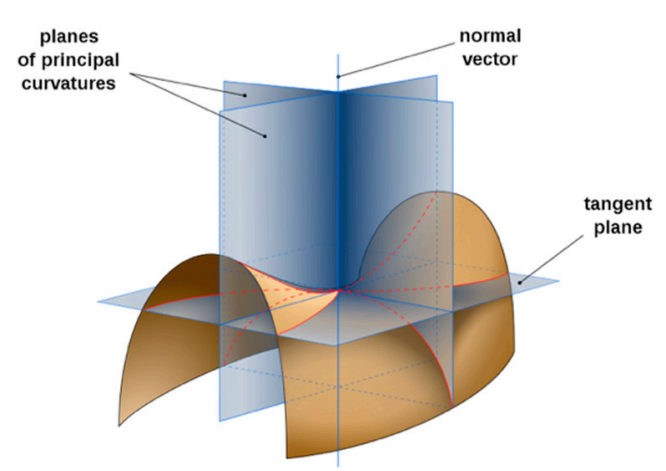

(b)

Figure 13. (a) Flat surface-based cellular material, screenshot from nTopology's Element [21], and (b) a saddle shaped surface showing minimal surface curvature planes and how a convex and concave curvature result in a mean curvature of zero at every point (Attr: Eric Gaba, Wikimedia Commons).

A particular manifestation of curved surfaces is the use of minimal surfaces, which have an average curvature of zero at every point on the surface. Figure $13 \mathrm{~b}$ shows two planes of principal curvature, that intersect the surface in question in two curves whose curvatures are equal and opposite, which is true at every point of minimal surfaces. Minimal surfaces are energetically favorable ways of weaving surfaces through space and mathematicians have developed several of these surfaces, some of which are discussed in a review article by Han et al. [52].

\subsection{Connectivity}

Once the type of tessellation is chosen and the elements are specified, the last step is to integrate the two. This is done by specifying a series of nodal co-ordinates and establishing connectivity between 
them. Alternatively, the designer can deploy an equation that assigns these to the unit cell, a method commonly used for surface-based cellular materials. For beam-based cellular materials, examples are shown in Figure 14 for cubic tessellations using beam elements, but with different nodes and connectivity. Connectivity can be between vertices at the corners of the tessellation, or even connect vertices along edges or faces. This form of connectivity can also be used to define surfaces between edges, one example of which is shown in Figure 13a.

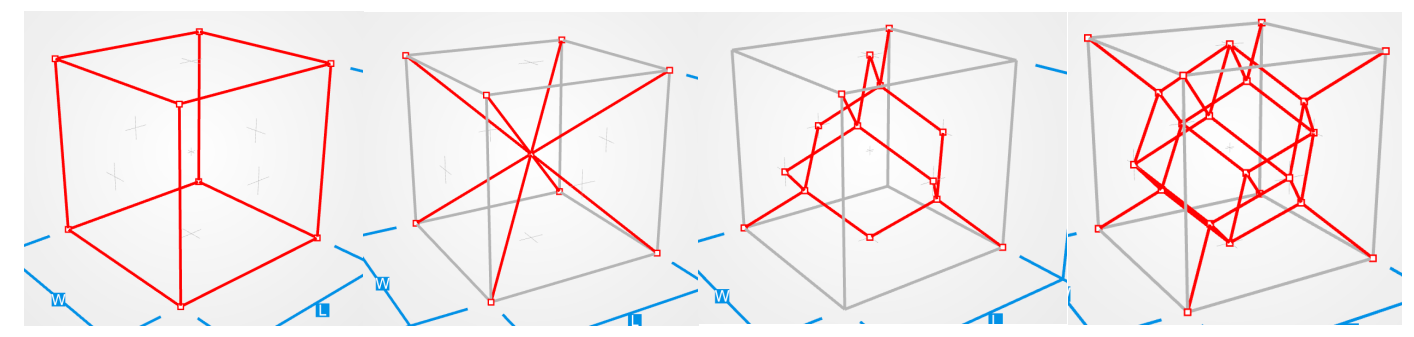

Figure 14. For a given tessellation and element, there are several ways to establish connectivity, as shown here for a cubic, beam element unit cell (screenshots from nTopology Element software [21]).

With regard to the previously discussed minimal surfaces, the surfaces of interest from a space-filling perspective are called Triply Periodic Minimal Surfaces (TPMS). First dating back to 1865, there are now several types of TPMS structures [53], a selection of which are shown in Figure 15. TPMS structures are developed from governing equations that can be implemented in CAD software, but interestingly, they also occur in nature and have been found both in living creatures (sea urchins, the scales on butterfly wings and beetle exoskeletons), as well as in zeolite and some liquid crystals [52].
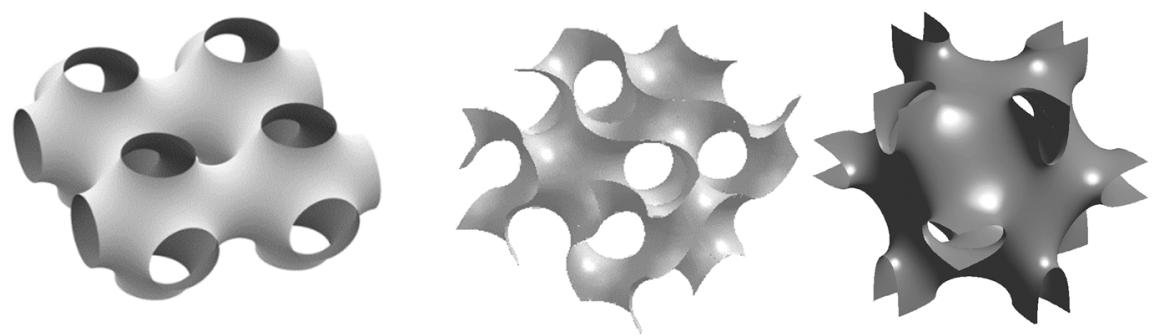

Figure 15. A selection of triply periodic minimal surfaces, left to right: the Schwarz P Surface, the Gyroid and the Neovius' surface (Attr, in order: Anders Sandberg, Catsquisher, Anders Sandberg, Wikimedia Commons).

\section{A Biomimetic Approach to Cellular Material Selection}

Each node in the classification scheme in Figure 8 represents a decision a designer needs to make. At the highest level, these decisions have to deal with selecting a strategy for tessellation, deciding what type of elements to use, and finally, deciding how to connect these elements within the tessellated unit cell. This section proposes that a biomimetic approach is ideal to help select the best tessellation strategy, as well as inform the selection of beam or surface elements. Traditional engineering methods can then be leveraged to define a connectivity for the identified elements using analytical, computational and/or experimental techniques. Most of the applied work in cellular material design tends to focus on selection of a connectivity scheme but forgoes addressing the first two aspects of the design classification in sufficient detail, based on generalizations such as bending-or stretch-dominated structures.

\subsection{Tessellation}

The first decision a designer encounters along the classification scheme in Figure 8 is whether to tessellate space using a periodic, stochastic or hierarchical scheme. All three strategies are observed 
in nature, and some examples of each are compiled in Table 3, along with the functions typically attributed to these structures in the literature.

\subsubsection{Periodic Tessellation}

Periodicity in natural cellular materials is most commonly observed in the nests of the social insects, such as the honeybee and the paper wasp [54]. This is not surprising, since the cells in these nests have a clear storage function, where each cell stores materials of a similar total volume; in the case of the honeybee, this is the storage of brood, pollen or nectar. The design of these nests is optimized to use material in efficient ways by forming hexagonal cells that have been shown mathematically to be the most efficient way of tessellating 2D space into partitions of equal area [55]. Periodic tessellations are also found in natural structures that do not have a storage function. Several radiolarian demonstrate periodic structures [56], as do the tesserae in rays [57] and scales on a wide range of fish and reptiles. For two-dimensional or planar tessellation in protective structures, a key function is to break up a continuous surface into inter-locking tiles that are less prone to fracture, while also providing flexibility, often by the use of a secondary material that is softer than the primary structural component, as discussed by Fratzl et al. [58].

In addition to serving a protective function against the external environment, these examples are all constructed around axes or planes of symmetry at the level of the organism. The radiolarian shell in Table 3, for example, has 3D radial symmetry. Scales on snakes and fish are constructed around a plane of bilateral symmetry. Stochastic patterns that have a mechanical function and are distributed about an axis or plane of symmetry could potentially violate this symmetry and its associated benefits, in particular for motor function [59].

In Figure 8, periodic tessellation is divided into two further decision points: one relates to the type of periodicity and the other the relationship between the periodic elements. A biological structure that embodies both these aspects is the pattern of scales on a snake. The scales on a snake have several functions: they provide a protective cover, minimize friction [60], provide flexibility and minimize moisture loss. However, the size of these scales varies-in particular, the ventral (underlying) region of the snake has long bands of scales as shown in Figure 16a, whereas the dorsal (top) side and the head and tail regions have smaller scales, as shown in Figure 16b.

The majority of periodic cellular materials may thus have one or more of the following conditions underlying their periodicity: (i) a storage function; (ii) use of a stiff-soft material hierarchy for fracture resilience; and/or (iii) a need to form around axes or planes of symmetry at the level of the organism. This raises the question: why bother with non-periodic tessellations at all?

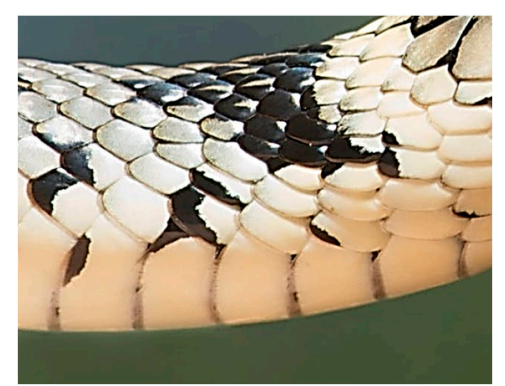

(a)

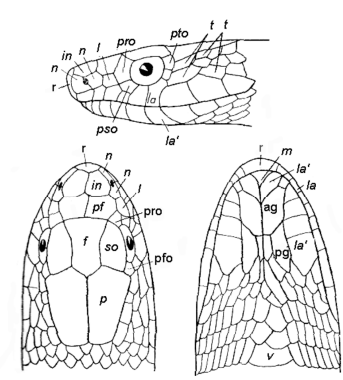

(b)

Figure 16. (a) Scales on a snake show smaller scale sizes on the top/dorsal side and larger bands of scales on the bottom/ventral side, minimizing frictional losses; (b) the head region shows a distribution of smaller scale sizes, probably adapted for flexibility to enable the jaw to open wide. 
Table 3. Natural examples of periodic, stochastic and branching tessellation.

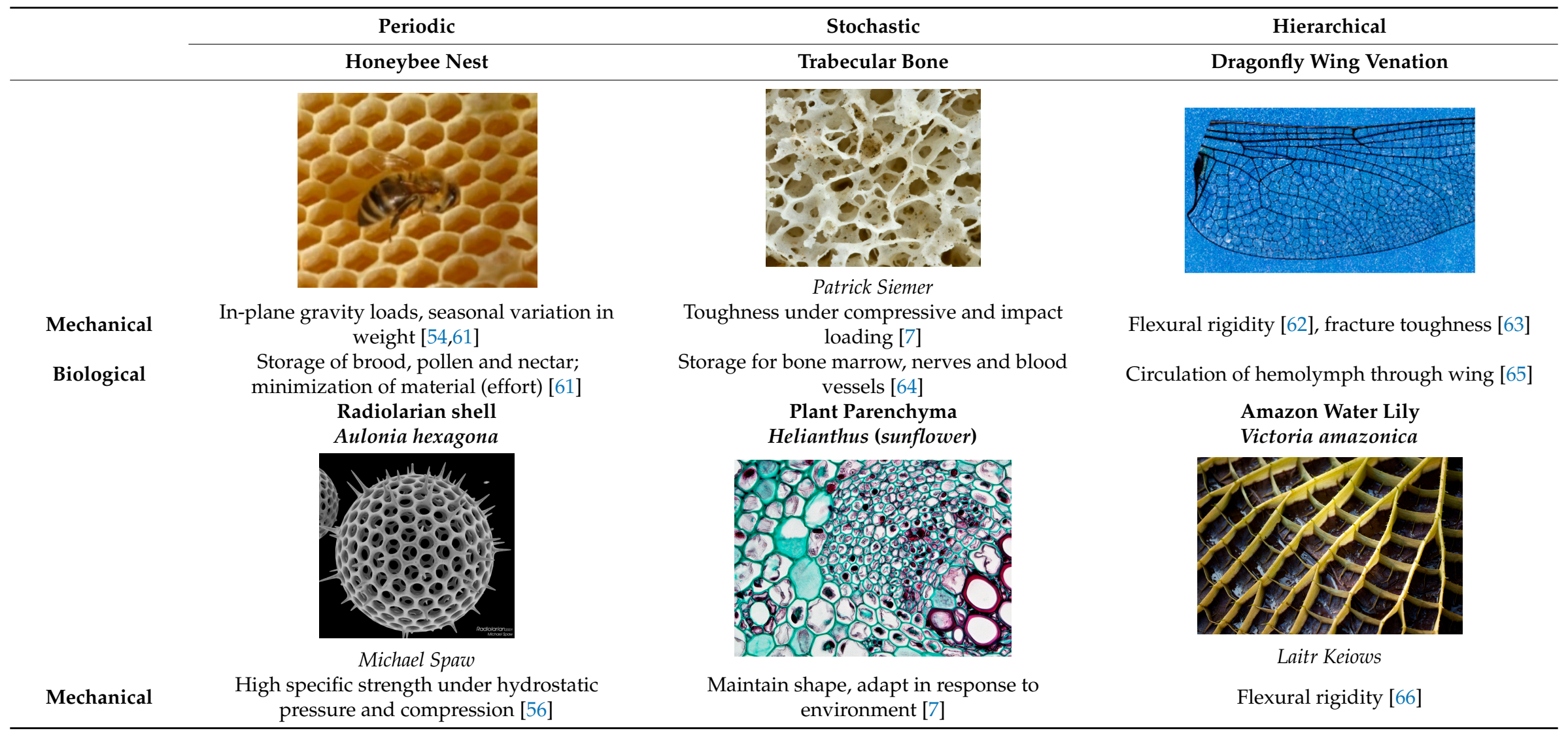


Table 3. Cont.

\begin{tabular}{|c|c|c|c|}
\hline & Periodic & Stochastic & Hierarchical \\
\hline & Honeybee Nest & Trabecular Bone & Dragonfly Wing Venation \\
\hline \multirow[t]{3}{*}{ Biological } & $\begin{array}{l}\text { Protective case, light-weight to occupy surface } \\
\text { waters [56] }\end{array}$ & Storage of sugars, photosynthesis [7] & Carry nutrients and waste products \\
\hline & Elasmobranchii endoskeleton tesserae & Veiled Lady indusium Phallus indusiatus & $\begin{array}{l}\text { Orange fruit } \\
\text { Hesperidium }\end{array}$ \\
\hline & Mason Dean (rights reserved), adapted from [57] & 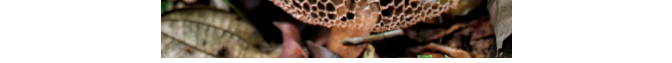 & \\
\hline Mechanical & $\begin{array}{l}\text { High bending flexibility; Compressive strength } \\
\text { [67] }\end{array}$ & Strength under variable loading (speculated) & Resilience under drop impact (speculated) \\
\hline Biological & $\begin{array}{l}\text { Space between tesserae provides room for } \\
\text { growth [57] }\end{array}$ & $\begin{array}{c}\text { Access for insects of varying sizes to fungal cap } \\
\text { (speculated) }\end{array}$ & Improve seed dispersal (speculated) \\
\hline
\end{tabular}




\subsubsection{Stochastic Tessellation}

Some structural cellular materials in nature do not follow the periodic regularity of structures such as the honeybee nest. This lack of periodicity is what is termed here as stochastic, whether these emerge from an underlying mathematical function (such as those resulting in the Voronoi pattern), or not. The breaking of symmetry in biology has received increasing interest of late, summarized in a review article by $\mathrm{Li}$ and Bowerman [68], who recognize that symmetry breaking occurs at various scales from molecular assemblies to embryonic body axes, and that it is intimately linked to functional diversification. This is well understood for molecular scales and for some examples of interest at larger scales, such as trabecular bone, shown in Table 3. Trabecular bone does indeed have to perform multiple functions: storage of bone marrow, nerves and blood vessels, as well as possess toughness under compressive and impact load [7]. Bone tissue also undergoes active strain-adaptive remodeling to allocate material where it is needed and remove it from where it is not [64]. This requirement to adapt to local strain inherently breaks symmetry since the interactions between the environment and the body do not result in a symmetric state of stress. Furthermore, this state of stress changes as the individual ages or as the load-bearing environment changes. Additionally, trabecular bone does not have a local symmetry requirement since an individual bone is not inherently symmetric, nor is there a requirement of equal storage volume within each cellular region.

Another example of stochastic patterns can be found in the ground tissue system in plants, which consists of parenchyma cells that store sugars and typically make up the bulk of non-woody plants. These cells can have very irregular shapes, as shown in Table 3, that while approaching a tetrakeidecahedron shape, have varying shapes and degrees of compaction. A final example of stochastic cellular materials is the pattern of the indusium of the Veiled Lady fungus, shown in Table 3. The functional benefits for this structure's stochastic nature is not clear, but since it serves as a network that enables several insect species to reach the fungal cap, it may be preferable to have a non-periodic pattern that can accommodate a wide range of insect sizes and mobility mechanisms.

Natural cellular materials, it may be speculated, tend to be stochastic when there is a need for one or more of the following conditions: (i) a high degree of multi-functionality; (ii) a need for the structure to be adaptive to changing loading conditions in the environment; and, (iii) a high degree of uncertainty in the magnitude and direction of these loads. This resonates well with what engineers have been practicing for decades with regard to energy-absorbing foams used in packaging, where the nature of the applied loads is uncertain.

While irregular cellular patterns suggest a lack of underlying relationships, empirical studies of natural stochastic cellular materials have revealed at least two relationships between cells in a certain neighborhood, which can then be generalized across a large area of interest. These two relationships are discussed briefly below and may form the basis of a stochastic design methodology for cellular materials.

- The Aboav-Weaire law: The Aboav-Weaire law relates the number of edges in a particular cell to the number of edges in the cells in its neighborhood. A general observation of stochastic cellular materials in nature is that a cell with more sides than average has neighbors which, when considered together, have fewer sides than the average number. For 2-dimensional honeycombs, this relationship was first given empirically by Aboav in 1970 (with a slightly different formulation) and derived formally by Weaire in 1974 [6] is:

$$
\bar{m}=5+\frac{6}{n}
$$

where $n$ is the number of edges of the candidate cell and $\bar{m}$ is the average number of edges of its $n$ neighbors (this is specific to edge-to-edge cells only). A fuller discussion of this law and its historical journey can be found in a review paper by Chiiu [69]. 
- The Lewis rule: F. T. Lewis studied cells in nature as a mathematical problem through the mid-1900s, publishing several papers in this field. The "Lewis rule" relates area of a cell to its number of edges [6]:

$$
\frac{A(n)}{A(\bar{n})}=\frac{n-n_{0}}{\bar{n}-n_{0}}
$$

where $A(n)$ is the area of a cell with $\mathrm{n}$ sides, $A(\bar{n})$ is that of the cell with average number of sides $\bar{n}$, and $n_{0}$ is a constant.

\subsubsection{Hierarchical Tessellation}

Cellular materials may also emerge around a hierarchy, such as a network of branches. This is especially true in the context of multi-functional design. One example of this is the venation patterns of the wing of the dragonfly. Veins in a dragonfly perform a circulatory function consistent with most branching patterns in nature, but also play a role in stiffening the vein $[62,70,71]$ and in increasing its resilience to damage [63]. A similar stiffening effect has been attributed to the veins of the Amazon water lily [66]. The key design relationships of branching involve the level of hierarchy, associated branch thickness and branching angles. For the latter two, the following empirical relationships have been proposed:

- Murray's law: This law, first proposed by Murray in 1926 [72], states that when a parent blood vessel branches into daughter vessels, the cube of the radius of the parent vessel is equal to the sum of the cubes of the radii of daughter blood vessels. This law has been generalized to fluidic networks of arbitrary shape and scale where for large scale channels, the ratio of the cross-sectional areas of the daughter-to-parent channels is equal to $N^{-2 / 3}$ where $N$ is the number of daughter channels (of constant cross-sectional shape) [73].

- Determination of Vertex angles: Thomson [74] studied the angles at the intersection of different veins and postulated that the angle was related to the "tensions" experienced in the veins. As shown in Figure 17, for a dragonfly wing, right angles $\left(90^{\circ}\right)$ are formed at the intersection of a thick, primary rib (presumably bearing more load) and a thin vein (with less load). Between these primary branches, the thin veins tend to meet at $120^{\circ}$ angles. Recent work on dragonfly venation has added more weight to this hypothesis, with data showing a similar relationship between the interior angles in a cell and the number of primary veins it is bounded by [43].

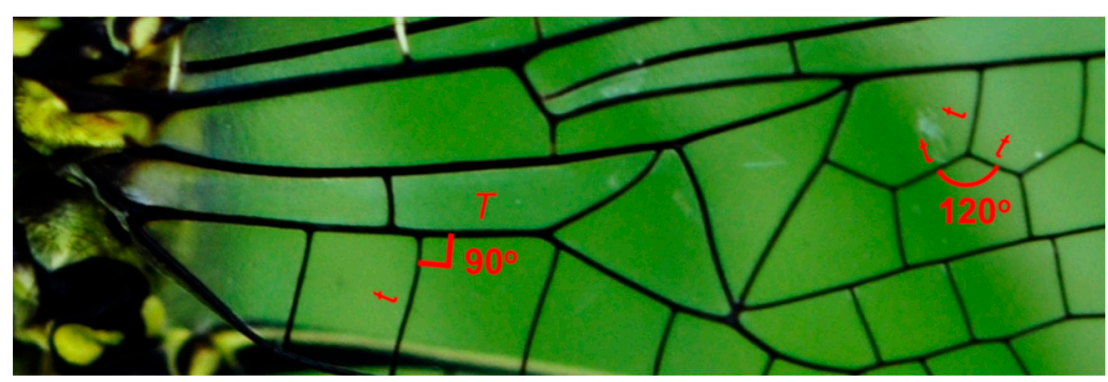

Figure 17. Thompson [75] postulated that when the "tensions" in veins had large differences $(\mathrm{T}>\mathrm{t})$, the veins would meet at right angles, approaching $120^{\circ}$ as the tensions in coincident veins equaled each other.

Not all hierarchical patterns have to physically manifest as branches-some may be the result of segmentation, such as the packing of cells in a citrus fruit as shown in Table 3, where an overlying high-level distribution into sections then influences the distribution of cells within each section. Another example of hierarchy is visible in the structure of the Venus flower basket, a sea sponge that has a silica lattice structure with multiple levels of cellularity, combining nested and overlaid 
strategies [75] for increased resilience to fracture. It is probable that most, if not all hierarchical tessellation emerges from the need for the cellular structure to address more than one function.

\subsection{Elements}

The majority of cellular materials that have found engineering application have been honeycombs and stochastic closed-cell foams, both shown previously in Figure 7. More recently, the growth in Additive Manufacturing and design capabilities has greatly increased interest in lattice materials. All three of these geometries employ one, or a combination of, two types of elemental features: beams or surfaces. Honeycombs consist of a pattern extruded in the out-of-plane direction, and foams resemble closely packed soap bubbles, and both are surface-based cellular structures. On the other hand, lattices are beam-based cellular structures.

Table 4 shows examples representing each of the three options discussed above. These and other natural cellular material examples suggest that nature tends to use surface elements either when resorting to some storage function (sugars for plants, larvae for nests) or for providing physical protection and acting as an external skin cover. When there is no storage or external protection requirement, most natural cellular materials are constructed from beams, as shown in the lattice structure of the Venus flower basket and the radiolarian, although more data is needed to confirm the validity of this observation.

Table 4. Examples of beam and surface elements in nature.

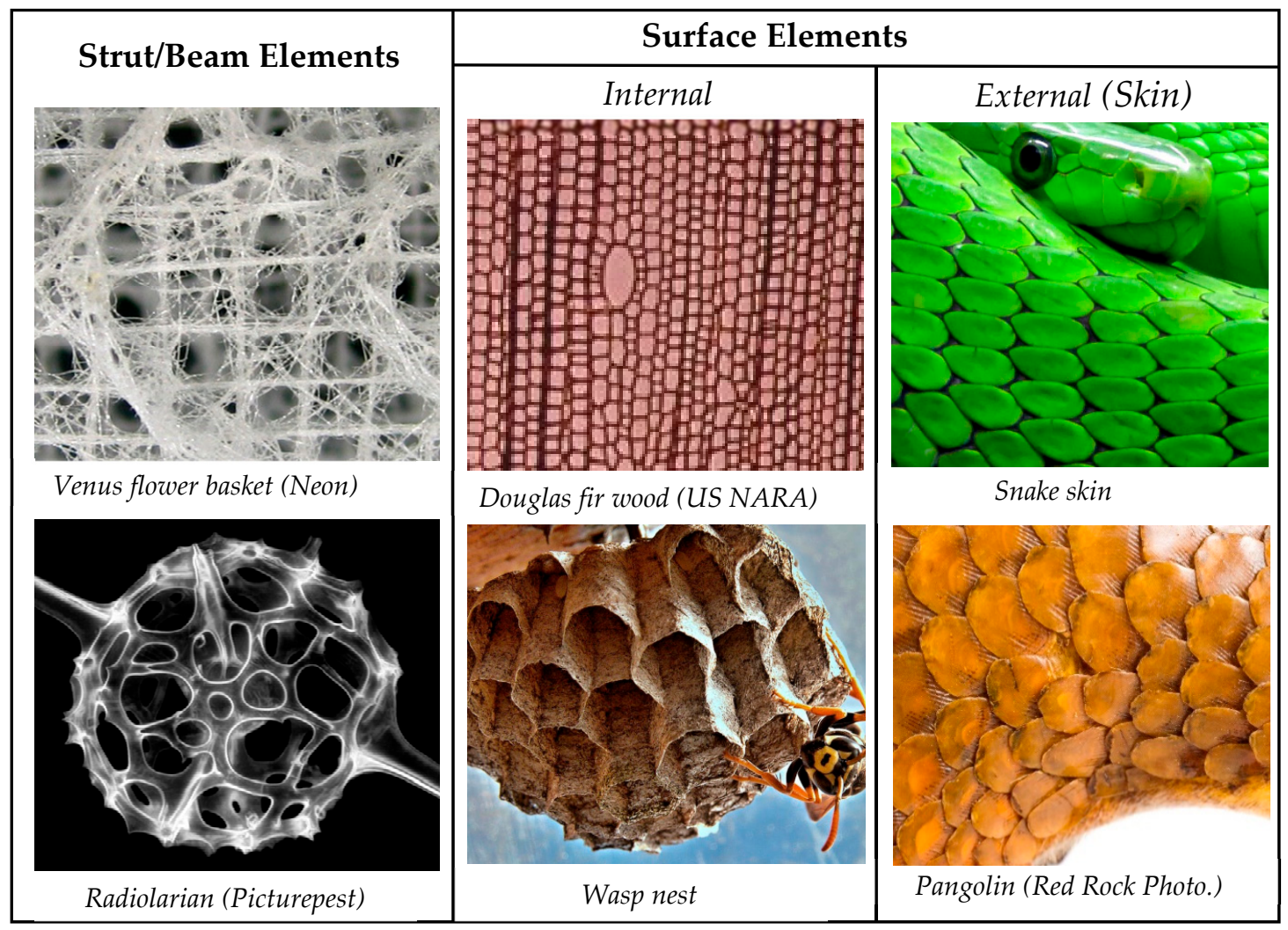

\subsection{Connectivity}

Unlike the previous two levels of the classification (tessellation and elements), there are infinitely many choices when it comes to the decision of connectivity. To narrow down the window of selection, the specific function(s) of interest needs to be articulated in order to identify natural models that perform that function [76]. 


\subsubsection{Functional Specification}

A common technique is to pose this as a question: "How would nature design for X function?" This paper is limited in scope to the design of load-bearing structures, where the following mechanical properties are of interest:

- Stiffness/rigidity (the ability to resist deformation),

- Strength (the ability to resist collapse), and

- Toughness (the ability to resist fracture)

These properties are defined in the context of the conditions experienced by the structure. With regard to the nature of loads, these may be classified as gravity loading, compression, tension, torsion, bending and shear, or some combination of these. Furthermore, these loads may be applied in one or many directions (such as uniaxial, biaxial and hydrostatic), and with different durations (such as at varying strain rates, fatigue and vibration). Thus, the loading condition may be adequately described by specifying three pieces of information (see Figure 18): loading type, loading direction, and period of application [9].

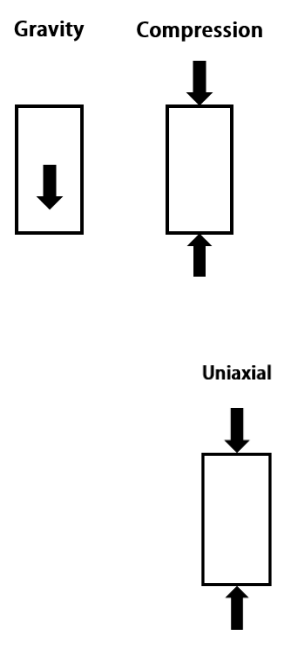

Monotonic

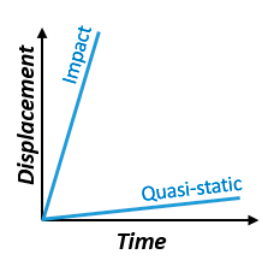

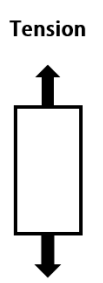
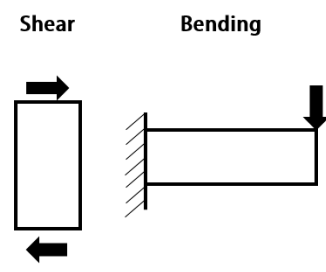

(a)

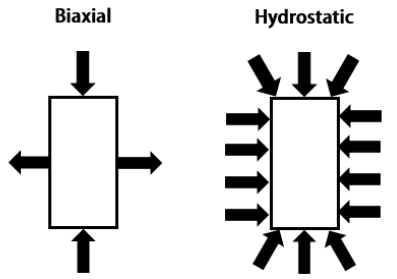

(b)

Creep

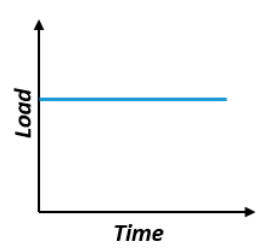

(c)
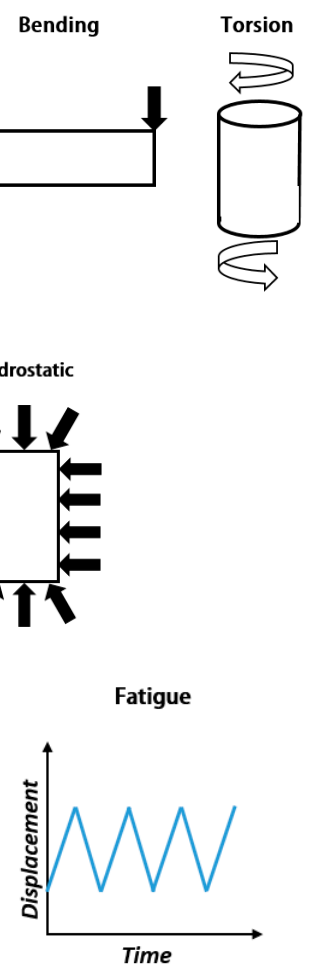

Figure 18. A proposed classification for loading conditions: (a) loading type, (b) loading direction and (c) period of application, first published by the authors in [9].

\subsubsection{Natural Models}

This approach, while simplifying from an application standpoint, increases the likelihood of finding studies in the literature of natural models that thrive in these conditions, whether through a formal review of the literature, or using online resources like AskNature.org [77]. Table 5 shows how, for eight identified natural cellular materials with an evident load bearing functional requirement, the loading conditions can be identified within the framework in Figure 18, and the relevant design choices described in Figure 8 may be extracted for each cellular material. Such a methodology is in principle extendable to a range of structural cellular materials and also to non-structural functions such as storage, thermal regulation, and acoustic modulation. 
Table 5. Biomimetic cellular materials design classification levels by loading conditions

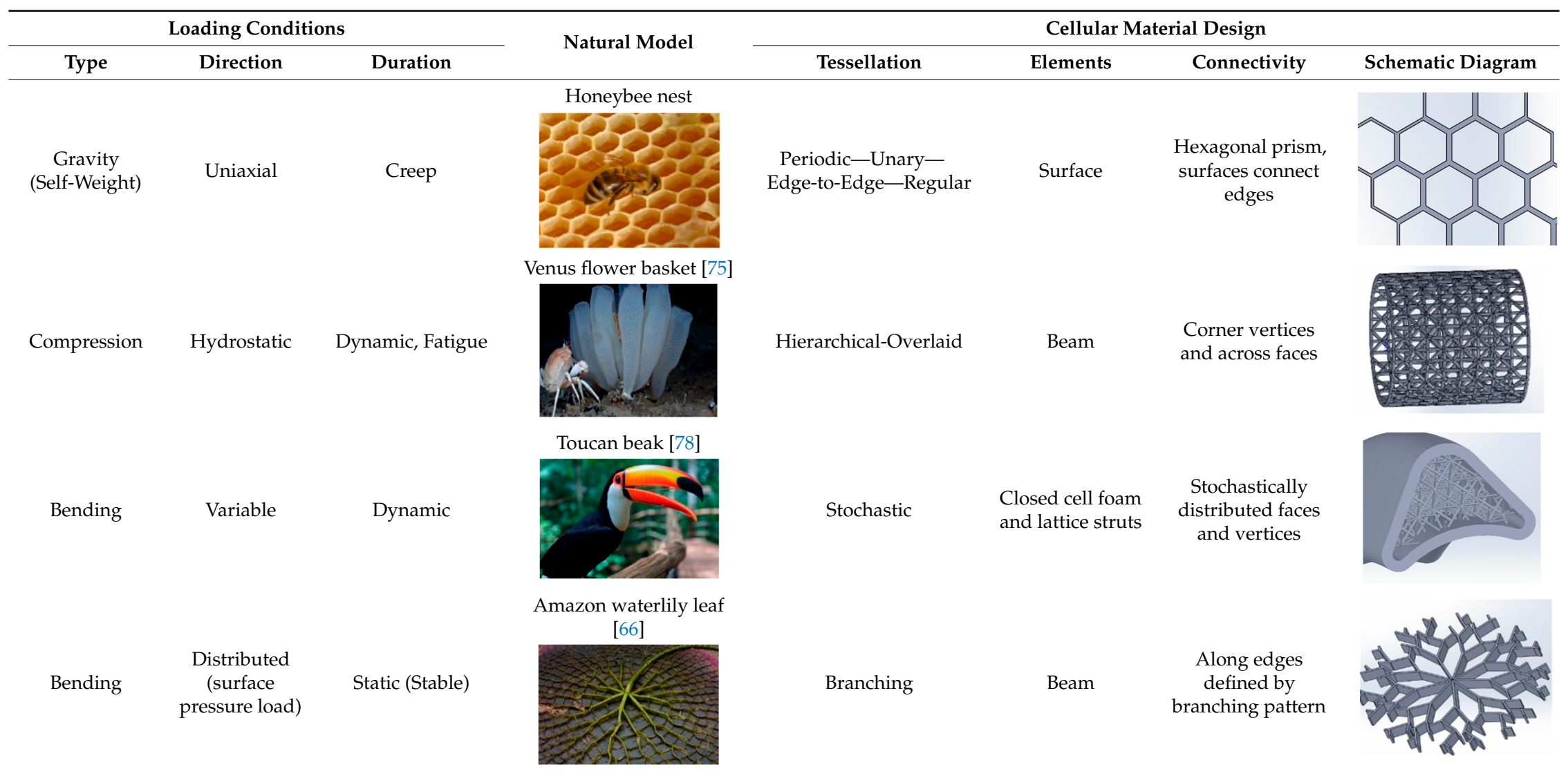


Table 5. Cont.

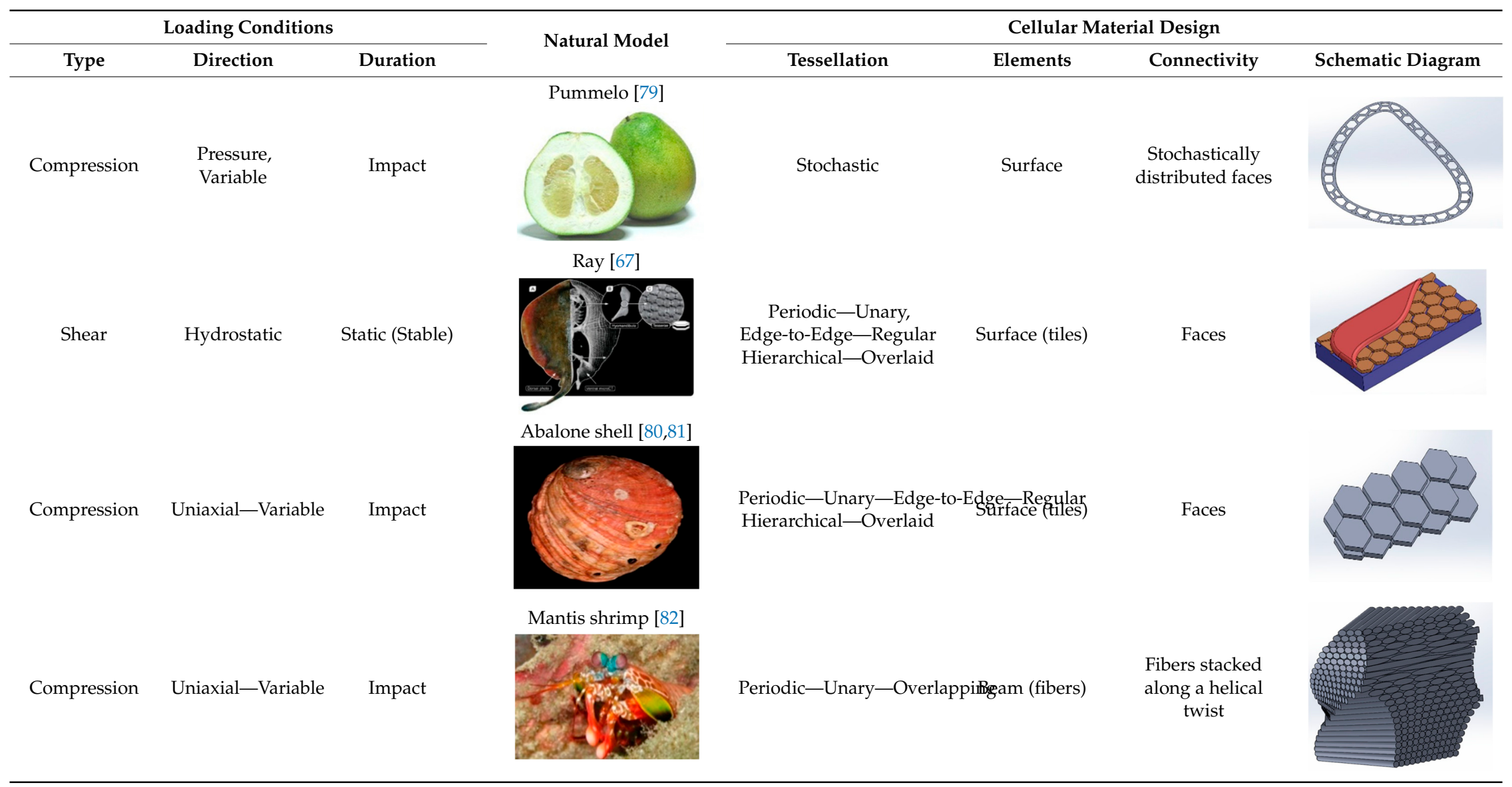


Connectivity is the last decision in the process of cellular material selection, and it specifies the actual shape of the unit cell. In that sense, this step provides the complete solution to the question of selection of a specific shape. The reason this step is not sufficient is that the previous decisions (tessellation and elements) can often provide functional insight that this final level of connectivity may not. Consider the hexagonal honeycomb of the honeybee, for example: a focus on wall-based connectivity along the edges of a hexagon for a self-supporting structure under gravity loads is not a strong enough reason to use this pattern in every application. One may ask as to why the tessellation is hexagonal (as opposed to stochastic or hierarchical), or why the structure is composed of surfaces (walls) instead of beams, and get to the underlying biological and structural benefits for these forms. For example, the hexagon is the best way of sub-dividing two-dimensional space into regions of equal area, something that has been mathematically proven [55]. However, the need for equal area (in reality, honeybee nests do not strictly have cells of equal area [61], but one may make this assumption for the present discussion) may not be relevant-in the bee's honeycomb, these cells serve a storage function that may not be of interest in the engineering application. For the same reason, honeybee nests need walls instead of just lattice-like struts. This is the reason a full comprehension of the design space must include an examination of cellular materials at all three levels: tessellation, elements and connectivity, and not just the last level alone.

\section{Discussion}

In this paper, we proposed a classification scheme for cellular materials from a designer's perspective. This classification scheme suggests three levels of decisions: tessellation, elements and connectivity, which when considered together, are sufficient to describe most unit cell designs. Engineering approaches that enable selection of cellular materials include analytical methods such as Maxwell's stability criterion and relative density-based scaling laws, as well as empirical methods such as experimentation and simulation. Analytical tools, while powerful in the insight they provide, are often restricted to simple shapes. Empirical methods can address complexity, but come with significant cost and time penalties, and do not provide much direct insight into the circumstances that make one cellular material (e.g., stochastic) preferable to another (e.g., periodic). We propose that natural cellular materials be considered as part of a guiding approach for cellular material selection in design.

\subsection{Design Methodology}

A mechanical designer seeking to leverage cellular materials may well ask how best to reconcile engineering and biomimetic approaches. A "biomimicry thinking" methodology has been proposed for those seeking to emulate solutions from nature, wherein a challenge may be posed to biology ("challenge to biology"), or natural models may be first discovered in nature and opportunities identified ("biology to design") — both approaches are shown graphically in Figure 19 [76].

These approaches involve four steps: scoping, discovering, creating, and evaluating and the reader is advised to study the cited reference to learn more.

For the specific problem of selecting a cellular material for a specific function, we propose the following approach inspired by the four steps in the "challenge to biology" methodology and shown graphically in Figure 20 for a specific example of structural honeycomb:

- $\quad$ Scoping: In this phase, the application context is identified and specific details of the environment (temperature, pressure, etc.) are also obtained. The functional requirements are then defined and understood to be valid in these contexts. In Figure 20, for example, a context could be aircraft paneling, where honeycomb structures are used to distribute stresses evenly.

- Discovering: Following this, natural models are identified that perform these functions in similar environments-insect nests, in this example. Importantly, measurements are then made on the natural model to abstract parameters and relationships between these parameters that may, in the next step, lead to design principles. 
- Creating: Design principles are developed to translate the abstracted biological principle into something of use to the engineering designer, which may result in an analytical and/or numerical model that can then be exercised. In this example, the role of the corner radius is examined, and a model is developed that incorporates it.

- $\quad$ Evaluating: In the final phase, the design principle is reconciled against other models, both in engineering and nature. We can use numerical and experimental techniques to validate our hypothesized design principle that the corner radius optimizes for specific stiffness, for example, and reconcile our findings with other insect nests and methods, such as scaling laws.
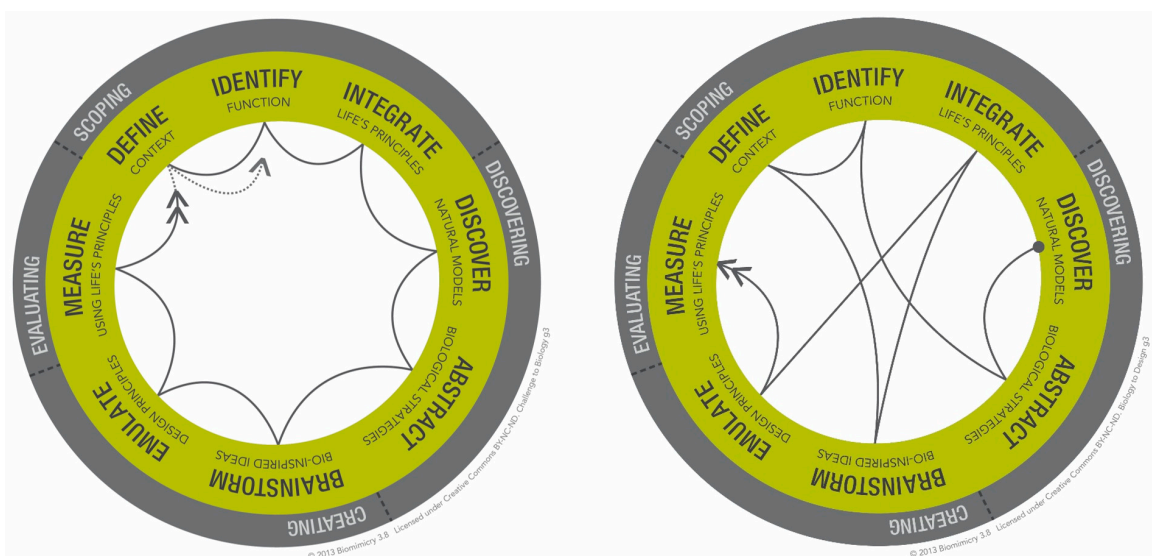

Figure 19. Biomimicry thinking methodology, as proposed in [76], showing the "challenge to biology" method on the left and the "biology to design" method on the right. Attribution: Biomimicry 3.8, creative commons BY-NC-ND.

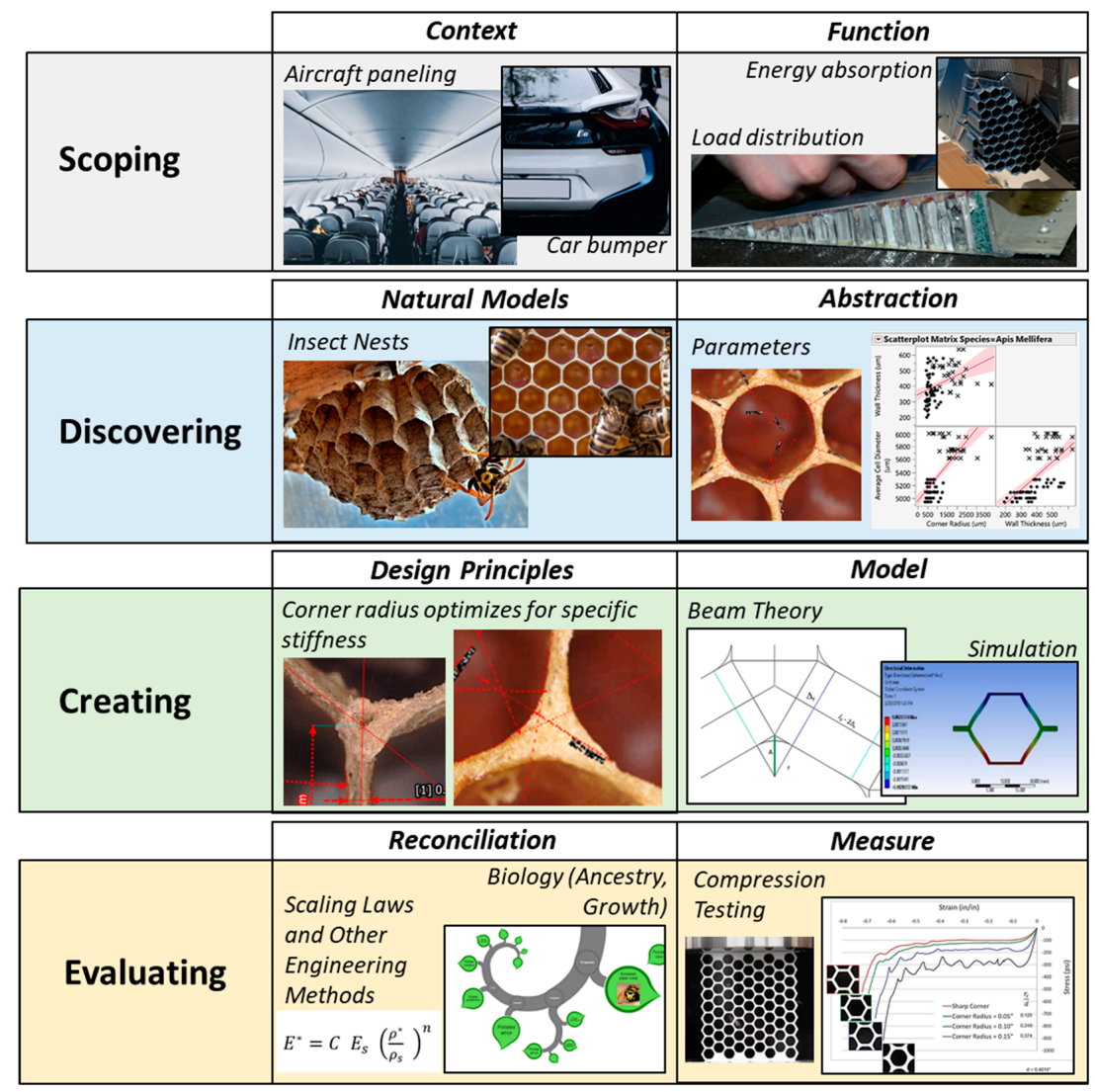

Figure 20. Incorporation of biomimicry thinking into the selection of cellular material designs, along with an example for the use of honeycomb designs in mechanical structures. Phylogenetic tree from OneZoom.org. 


\subsection{Limitations of Study}

In addition to the limited scope of this work, restricted as it is to structural parameters only, this work is also limited to the identification of a specific unit cell design. While this is arguably the central question in cellular materials design, it is not the only one. Other questions include:

- $\quad$ How should the density of the cellular material be prescribed within the structure? Should it be completely full of cellular materials, or are there benefits to including empty (or negative) space [83]?

- How should local parameters such as thickness, beam shape be optimized? and

- What is the optimum termination strategy for the cellular material at the boundary of the structure?

A second limitation of the study is that, for the most part, it ignores discussion of the contributions of ancestry (phylogeny) and development (ontogeny) to the resulting form. We assume, therefore, that the observed structure is optimal for the function at hand and that any constraints that phylogeny and ontogeny have placed on it do not detract from the inherent benefit of that structure relative to the function of interest. Finally, while this paper does develop a new classification scheme and demonstrate a methodology for studying natural cellular materials relative to this scheme, we concede that much work is needed in order to implement biomimetic cellular materials as a considered option in mechanical design beyond just the conceptual design phase. A framework such as the one proposed here has the potential to further accelerate discovery of biomimetic cellular materials and their application in design.

Author Contributions: Conceptualization, D.B., C.A.P., L.A.F. and C.L.; Methodology, D.B.; Writing-Original Draft Preparation, D.B.; Writing-Review \& Editing, D.B., C.A.P., C.L. and L.A.F.; Funding Acquisition, D.B., L.A.F. and C.L.

Funding: This research was partially funded by a seed grant awarded by the Biomimicry Center at Arizona State University.

Acknowledgments: The authors would like to acknowledge Raghav Sharma for providing the simulation results shown in Figure 6, and Austin Suder for CAD schematics in Table 5, both students at Arizona State University at the time of writing this paper.

Conflicts of Interest: The authors declare no conflict of interest.

\section{References}

1. Ball, P. Shapes: Nature's Patterns: A Tapestry in Three Parts; OUP Oxford: Oxford, UK, 2009.

2. Schaedler, T.A.; Carter, W.B. Architected Cellular Materials. Annu. Rev. Mater. Res. 2016, 46, 187-210. [CrossRef]

3. Ashby, M.F.; Evans, A.G.; Fleck, N.A.; Gibson, L.J.; Hutchinson, J.W.; Wadley, H.N.G. Metal Foams: A Design Guide; Butterworth Heinemann: Oxford, UK, 2000.

4. Tashiro, T.; Ishida, A.; Hori, M.; Igisu, M.; Koike, M.; Méjean, P.; Takahata, N.; Sano, Y.; Komiya, T. Early trace of life from 3.95 Ga sedimentary rocks in Labrador, Canada. Nature 2017, 549, 516-518. [CrossRef] [PubMed]

5. Varro, M.T. Rerum Rusticarum Libri III; Oxford University Press: London, UK, 1800.

6. Gibson, L.J.; Ashby, M.F. Cellular Solids: Structure and Properties, 2nd ed.; Cambridge Solid State Science Series; Cambridge University Press: Cambridge, UK, 1999.

7. Gibson, L.J.; Ashby, M.F.; Harley, B.A. Cellular Materials in Nature and Medicine, 1st ed.; Cambridge University Press: Cambridge, UK, 2010.

8. du Plessis, A.; Broeckhoven, C. Looking deep into nature: A review of micro-computed tomography in biomimicry. Acta Biomater. 2019, 85, 27-40. [CrossRef] [PubMed]

9. Mcnulty, T.; Bhate, D.; Zhang, A.; Kiser, M.A.; Ferry, L.; Suder, A.; Bhattacharya, S.; Boradkar, P. A Framework for the Design of Biomimetic Cellular Materials for Additive Manufacturing. In Proceedings of the 28th Annual International Solid Freeform Fabrication Symposium, Austin, TX, USA, 7-9 August 2017; pp. 2188-2200. 
10. Ashby, M.F. Materials Selection in Mechanical Design, 4th ed.; Butterworth-Heinemann, Elsevier: Amsterdam, The Netherlands, 2011.

11. Berger, J.B.; Wadley, H.N.G.; McMeeking, R.M. Mechanical metamaterials at the theoretical limit of isotropic elastic stiffness. Nature 2017, 543, 533-537. [CrossRef] [PubMed]

12. Maxwell, J.C. On reciprocal figures and diagrams of forces. Philos. Mag. 1864, 4, 250-261. [CrossRef]

13. Deshpande, V.S.; Ashby, M.F.; Fleck, N.A. Foam topology: Bending versus stretching dominated architectures. Acta Mater. 2001, 49, 1035-1040. [CrossRef]

14. O'Masta, M.R.; Dong, L.; St-Pierre, L.; Wadley, H.N.G.; Deshpande, V.S. The fracture toughness of octet-truss lattices. J. Mech. Phys. Solids 2017, 98, 271-289. [CrossRef]

15. Gümrük, R.; Mines, R.A.W. Compressive behaviour of stainless steel micro-lattice structures. Int. J. Mech. Sci. 2013, 68, 125-139. [CrossRef]

16. Meza, L.R.; Phlipot, G.P.; Portela, C.M.; Maggi, A.; Montemayor, L.C.; Comella, A.; Kochmann, D.M.; Greer, J.R. Reexamining the mechanical property space of three-dimensional lattice architectures. Acta Mater. 2017, 140, 424-432. [CrossRef]

17. Yan, C.; Hao, L.; Hussein, A.; Young, P.; Huang, J.; Zhu, W. Microstructure and mechanical properties of aluminium alloy cellular lattice structures manufactured by direct metal laser sintering. Mater. Sci. Eng. A 2015, 628, 238-246. [CrossRef]

18. Yu, X.; Zhou, J.; Liang, H.; Jiang, Z.; Wu, L. Mechanical metamaterials associated with stiffness, rigidity and compressibility: A brief review. Prog. Mater. Sci. 2018, 94, 114-173. [CrossRef]

19. Beyer, C.; Figueroa, D. Design and analysis of lattice structures for additive manufacturing. J. Manuf. Sci. Eng. 2016, 138, 121014. [CrossRef]

20. Zhang, P.; Toman, J.; Yu, Y.; Biyikli, E.; Kirca, M.; Chmielus, M.; To, A.C. Efficient design-optimization of variable-density hexagonal cellular structure by additive manufacturing: Theory and validation. J. Manuf. Sci. Eng. 2015, 137, 021004. [CrossRef]

21. NTopology. Element; NTopology: New York, NY, USA, 2018.

22. Sigmund, O.; Aage, N.; Andreassen, E. On the (non-)optimality of Michell structures. Struct. Multidiscip. Optim. 2016, 54, 361-373. [CrossRef]

23. Osanov, M.; Guest, J.K. Topology Optimization for Architected Materials Design Keywords. Annu. Rev. Mater. Res. 2016, 46, 211-233. [CrossRef]

24. Cadman, J.E.; Zhou, S.; Chen, Y.; Li, Q. On design of multi-functional microstructural materials. J. Mater. Sci. 2013, 48, 51-66. [CrossRef]

25. Bendsoe, M.P.; Sigmund, O. Topology Optimization: Theory, Methods and Applications; Springer: Berlin/Heidelberg, Germany, 2003.

26. Sigmund, O. Design of Material Structures Using Topology Optimization. Ph.D. Thesis, Technical University of Denmark, Kongens Lyngby, Denmark, 1994.

27. Carstensen, J.V. Topology Optimization Algorithms for Improved Manufacturability and Cellular Material Design. Ph.D. Thesis, Johns Hopkins University, Baltimore, MD, USA, 2017.

28. Cheng, L.; Liu, J.; Liang, X.; To, A.C. Coupling lattice structure topology optimization with design-dependent feature evolution for additive manufactured heat conduction design. Comput. Methods Appl. Mech. Eng. 2018, 332, 408-439. [CrossRef]

29. Cheng, L.; Zhang, P.; Biyikli, E.; Bai, J.; Robbins, J.; To, A. Efficient design optimization of variable-density cellular structures for additive manufacturing: Theory and experimental validation. Rapid Prototyp. J. 2017, 23, 660-677. [CrossRef]

30. Da, D.C.; Chen, J.H.; Cui, X.Y.; Li, G.Y. Design of materials using hybrid cellular automata. Struct. Multidiscip. Optim. 2017, 56, 131-137. [CrossRef]

31. Basanta, D.; Bentley, P.J.; Miodownik, M.A.; Holm, E.A. Evolving Cellular Automata to Grow Microstructures. In European Conference on Genetic Programming; Springer: Berlin/Heidelberg, Germany, 2003.

32. Gu, G.X.; Chen, C.-T.; Richmond, D.J.; Buehler, M.J. Bioinspired hierarchical composite design using machine learning: Simulation, additive manufacturing, and experiment. Mater. Horiz. 2018, 5, 939-945. [CrossRef]

33. Gu, G.X.; Chen, C.-T.; Buehler, M.J. De novo composite design based on machine learning algorithm. Extrem. Mech. Lett. 2018, 18, 19-28. [CrossRef] 
34. Zhang, Q.; Yang, X.; Li, P.; Huang, G.; Feng, S.; Shen, C.; Han, B.; Zhang, X.; Jin, F.; Xu, F.; et al. Bioinspired engineering of honeycomb structure-Using nature to inspire human innovation. Prog. Mater. Sci. 2015, 74, 332-400. [CrossRef]

35. Ashby, M. The properties of foams and lattices. Philos. Trans. R. Soc. A Math. Phys. Eng. Sci. 2006, 364, 15-30. [CrossRef] [PubMed]

36. Chavey, D. Tilings by Regular Polygons-II A Catalog of Tilings. Comput. Math. Applic. 1989, 17, 147-165. [CrossRef]

37. Møller, J.; Stoyan, D. Stochastic Geometry and Random Tessellations. Available online: http:/ / people.math. aau.dk/ \{\}jm/FinalJMDS.pdf (accessed on 17 March 2019).

38. van Lieshout, M.N.M. An introduction to planar random tessellation models. Spat. Stat. 2012, 1, 40-49. [CrossRef]

39. Zok, F.W.; Latture, R.M.; Begley, M.R. Periodic truss structures. J. Mech. Phys. Solids 2016, 96, $184-203$. [CrossRef]

40. Fazelpour, M. Developing Unit Cell Design Guidelines for Meso-Scale Periodic Cellular Materials. Ph.D. Thesis, Clemson University, Clemson, SC, USA, December 2016.

41. Lenngren, N. K-Uniform Tilings By Regular Polygons. Available online: http://citeseerx.ist.psu.edu/ viewdoc/download?doi=10.1.1.148.7628\&rep=rep1\&type=pdf (accessed on 17 March 2019).

42. Grünbaum, B.; Shepard, G. Tilings by Regular Polygons. Math. Mag. 1977, 50, 227-247. [CrossRef]

43. Hoffmann, J.; Donoughe, S.; Li, K.; Salcedo, M.K.; Rycroft, C.H. A simple developmental model recapitulates complex insect wing venation patterns. Proc. Natl. Acad. Sci. USA 2018, 115, 9905-9910. [CrossRef]

44. Pearce, P. Structure in Nature is a Strategy for Design, 5th ed.; The MIT Press: Cambridge, MA, USA, 1990.

45. Pearce, P.; Pearce, S. Polyedra Primer; Van Nostrand Reinhold Company: New York, NY, USA, 1979.

46. Bhate, D. Lattice Design Optimization: Crowdsourcing Ideas in the Classroom. In Proceedings of the 29th Annual International Solid Freeform Fabrication Symposium, Austin, TX, USA, 13-15 August 2018.

47. Contuzzi, N.; Campanelli, S.L.; Casavola, C.; Lamberti, L. Manufacturing and characterization of $18 \mathrm{Ni}$ marage 300 lattice components by selective laser melting. Materials 2013, 6, 3451-3468. [CrossRef]

48. Sing, S.L.; Yeong, W.Y.; Wiria, F.E.; Tay, B.Y. Characterization of Titanium Lattice Structures Fabricated by Selective Laser Melting Using an Adapted Compressive Test Method. Exp. Mech. 2016, 56, 735-748. [CrossRef]

49. Liu, J.; Gaynor, A.T.; Chen, S.; Kang, Z.; Suresh, K.; Takezawa, A.; Li, L.; Kato, J.; Tang, J.; Wang, C.C.; et al. Current and future trends in topology optimization for additive manufacturing. Struct. Multidiscip. Optim. 2018, 57, 2458-2483. [CrossRef]

50. Yang, L.; Hsu, K.; Baughman, B.; Godfrey, D.; Medina, F.; Menon, M.; Wiener, S. Additive Manufacturing of Metals: The Technology, Materials, Design and Production; Springer: Berlin/Heidelberg, Germany, 2017.

51. Queheillalt, D.T.; Deshpande, V.S.; Wadley, H.N.G. Truss waviness effects in cellular lattice structures. J. Mech. Mater. Struct. 2007, 2, 1657-1675. [CrossRef]

52. Han, L.; Che, S. An Overview of Materials with Triply Periodic Minimal Surfaces and Related Geometry: From Biological Structures to Self-Assembled Systems. Adv. Mater. 2018, 1705708, 1-22. [CrossRef] [PubMed]

53. Schoen, A.H. Reflections concerning triply-periodic minimal surfaces. Interface Focus 2012, 2, $658-668$. [CrossRef] [PubMed]

54. von Frisch, K. Animal Architecture (English and German Edition), 1st ed.; Harcourt: New York, NY, USA, 1974.

55. Hales, T.C. The honeycomb conjecture. Discret. Comput. Geom. 2001, 25, 1-22. [CrossRef]

56. Maier, M.; Hamm, C. ELISE 3D-A database-driven engineering and design tool. In Proceedings of the International Conference on Engineering Design, ICED11, Kongens Lyngby, Denmark, 15-18 August 2011.

57. Seidel, R.; Lyons, K.; Blumer, M.; Zaslansky, P.; Fratzl, P.; Weaver, J.C.; Dean, M.N. Ultrastructural and developmental features of the tessellated endoskeleton of elasmobranchs (sharks and rays). J. Anat. 2016, 229, 681-702. [CrossRef]

58. Fratzl, P.; Kolednik, O.; Fischer, F.D.; Dean, M.N. The mechanics of tessellations-bioinspired strategies for fracture resistance. Chem. Soc. Rev. 2016, 45, 252-267. [CrossRef]

59. Du Sautoy, M. Symmetry: A Journey into the Patterns of Nature; Harper Collins: New York, NY, USA, 2008.

60. Filippov, A.E.; Gorb, S.N. Modelling of the frictional behaviour of the snake skin covered by anisotropic surface nanostructures. Sci. Rep. 2016, 6, 23539. [CrossRef] 
61. Hepburn, H.R.; Pirk, C.W.W.; Duangphakdee, O. Honeybee Nests; no. 1929; Springer: Berlin/Heidelberg, Germany, 2014.

62. Jongerius, S.R.; Lentink, D. Structural Analysis of a Dragonfly Wing. Exp. Mech. 2010, 50, $1323-1334$. [CrossRef]

63. Dirks, J.H.; Taylor, D. Veins improve fracture toughness of insect wings. PLoS ONE 2012, 7, e43411. [CrossRef]

64. Allen, M.R.; Burr, D.B. Bone Modeling and Remodeling. In Basic and Applied Bone Biology; Academic Press: Cambridge, MA, USA, 2013; pp. 75-90.

65. Chintapalli, R.T.V.; Hillyer, J.F. Hemolymph circulation in insect flight appendages: Physiology of the wing heart and circulatory flow in the wings of the mosquito Anopheles gambiae. J. Exp. Biol. 2016, 219, 3945-3951. [CrossRef]

66. Nielsen, D. Victoria regia's bequest to modern architecture. Wit Trans. Ecol. Environ. 2010, 138, 65-76.

67. Liu, X.; Dean, M.N.; Summers, A.P.; Earthman, J.C. Composite model of the shark's skeleton in bending: A novel architecture for biomimetic design of functional compression bias. Mater. Sci. Eng. C 2010, 30, 1077-1084. [CrossRef]

68. Li, R.; Bowerman, B. Symmetry breaking in biology. Cold Spring Harb. Perspect. Biol. 2010, 2, a003475. [CrossRef]

69. Chiiu, S.N. Aboav-Weaire's and Lewis' Laws-A Review. Mater. Charact. 1995, 34, 149-165. [CrossRef]

70. Combes, S.A. Flexural stiffness in insect wings I. Scaling and the influence of wing venation. J. Exp. Biol. 2003, 206, 2979-2987. [CrossRef]

71. Kingslover, J.G.; Koehl, M.A.R. Aerodynamics, Thermoregulation, and the Evolution of Insect Wings: Differential Scaling and Evolutionary Change. Evolution 2016, 39, 488-504. [CrossRef]

72. Murray, B.C.D. The Physiological Principle of Minimum Work. I. The Vascular System and the Cost of Blood Volume. Proc. Natl. Acad. Sci. USA 1926, 13, 207-214. [CrossRef]

73. Stephenson, D.; Patronis, A.; Holland, D.M.; Lockerby, D.A. Generalizing Murray's law: An optimization principle for fluidic networks of arbitrary shape and scale. J. Appl. Phys. 2016, 118, 174302. [CrossRef]

74. Thompson, D.W. On Growth and Form, 1st ed.; Cambridge University Press: Cambridge, UK, 1917.

75. Aizenberg, J.; Weaver, J.C.; Thanawala, M.S.; Sundar, V.C.; Morse, D.E.; Fratzl, P. Materials science: Skeleton of Euplectella sp.: Structural hierarchy from the nanoscale to the macroscale. Science 2005, 309, 275-278. [CrossRef]

76. Baumeister, D. Biomimicry Resource Handbook: A Seed Bank of Best Practices; Biomimicry 3.8: Missoula, MT, USA, 2014.

77. The Biomimicry Institute. Ask Nature. Available online: https:/ /asknature.org/ (accessed on 7 May 2018).

78. Seki, Y.; Kad, B.; Benson, D.; Meyers, M.A. The toucan beak: Structure and mechanical response. Mater. Sci. Eng. C 2006, 26, 1412-1420. [CrossRef]

79. Fischer, S.F.; Thielen, M.; Loprang, R.R.; Seidel, R.; Fleck, C.; Speck, T.; Bührig-Polaczek, A. Pummelos as concept generators for biomimetically inspired low weight structures with excellent damping properties. Adv. Eng. Mater. 2010, 12, 658-663. [CrossRef]

80. Meyers, M.A.; Chen, P.Y.; Lopez, M.I.; Seki, Y.; Lin, A.Y.M. Biological materials: A materials science approach. J. Mech. Behav. Biomed. Mater. 2011, 4, 626-657. [CrossRef]

81. Lin, A.; Meyers, M.A. Growth and structure in abalone shell. Mater. Sci. Eng. A 2005, 390, 27-41. [CrossRef]

82. Patek, S.N.; Korff, W.L.; Caldwell, R.L. Deadly strike mechanism of a mantis shrimp. Nature 2004, 428, 819-820. [CrossRef]

83. Casanova, L.; Vijayan Anitha, V.; Kadway, N.; Gandhi, A.; Le, T.; Lee, C.; Bhate, D. On the Mechanical Behavior of Additively Manufactured Asymmetric Honeycombs. In Proceedings of the 29th Annual International Solid Freeform Fabrication Symposium, Austin, TX, USA, 13-15 August 2018.

(C) 2019 by the authors. Licensee MDPI, Basel, Switzerland. This article is an open access article distributed under the terms and conditions of the Creative Commons Attribution (CC BY) license (http:/ / creativecommons.org/licenses/by/4.0/). 